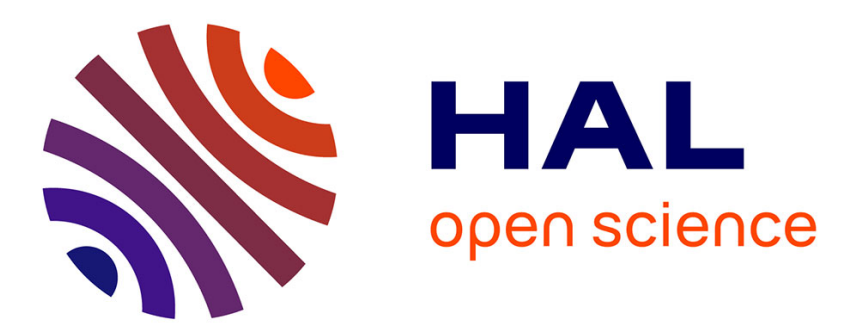

\title{
Stability Analysis of Optimal Control Problems with a Second-order State Constraint
}

\author{
Audrey Hermant
}

\section{To cite this version:}

Audrey Hermant. Stability Analysis of Optimal Control Problems with a Second-order State Constraint. SIAM Journal on Optimization, 2009, 20 (1), pp.104-129. 10.1137/070707993 . inria$00186968 \mathrm{v} 2$

\section{HAL Id: inria-00186968 https://hal.inria.fr/inria-00186968v2}

Submitted on 14 Nov 2007

HAL is a multi-disciplinary open access archive for the deposit and dissemination of scientific research documents, whether they are published or not. The documents may come from teaching and research institutions in France or abroad, or from public or private research centers.
L'archive ouverte pluridisciplinaire HAL, est destinée au dépôt et à la diffusion de documents scientifiques de niveau recherche, publiés ou non, émanant des établissements d'enseignement et de recherche français ou étrangers, des laboratoires publics ou privés. 
INSTITUT NATIONAL DE RECHERCHE EN INFORMATIQUE ET EN AUTOMATIQUE

Stability Analysis of Optimal Control Problems with a Second-order State Constraint

Audrey Hermant

$\mathbf{N}^{\circ}$ ????

November 2007

Thème NUM

apport

de recherche 



\title{
RINRIA
}

\section{Stability Analysis of Optimal Control Problems with a Second-order State Constraint}

\author{
Audrey Hermant* \\ Thème NUM — Systèmes numériques \\ Projets COMMANDS \\ Rapport de recherche $\mathrm{n}^{\circ}$ ???? — November 2007 - 33 pages
}

\begin{abstract}
This paper gives stability results for nonlinear optimal control problems subject to a regular state constraint of second-order. The strengthened Legendre-Clebsch condition is assumed to hold, and no assumption on the structure of the contact set is made. Under a weak second-order sufficient condition (taking into account the active constraints), we show that the solutions are Lipschitz continuous w.r.t. the perturbation parameter in the $L^{2}$ norm, and Hölder continuous in the $L^{\infty}$ norm. We use a generalized implicit function theorem in metric spaces by Dontchev and Hager [SIAM J. Control Optim., 1998]. The difficulty is that multipliers associated with second-order state constraints have a low regularity (they are only bounded measures). We obtain Lipschitz stability of a "primitive" of the state constraint multiplier.
\end{abstract}

Key-words: Optimal control, second-order state constraint, stability analysis, alternative formulation, sufficient second-order optimality condition, uniform quadratic growth, strong regularity.

* CMAP, École Polytechnique, INRIA Futurs, Route de Saclay, 91128 Palaiseau, France

Unité de recherche INRIA Futurs Parc Club Orsay Université, ZAC des Vignes, 4, rue Jacques Monod, 91893 ORSAY Cedex (France) Téléphone : +33172925900-Télécopie : +33160196608 


\section{Analyse de stabilité pour les problèmes de commande optimale avec une contrainte sur l'état du second-ordre}

Résumé : Dans cet article on donne un résultat de stabilité pour les problèmes de commande optimale avec une contrainte sur l'état du second-ordre régulière. La condition forte de Legendre-Clebsch est supposée satisfaite. Sous une condition suffisante du secondordre faible (prenant en compte les contraintes actives) on montre que les solutions sont lipschitziennes par rapport au paramètre pour la norme $L^{2}$, et höldériennes pour la norme $L^{\infty}$. On utilise un théorème des fonctions implicites généralisé dans des espaces métriques de Dontchev et Hager [SIAM J. Control Optim., 1998]. La difficulté vient du fait que les multiplicateurs associés aux contraintes sur l'état du second-ordre sont peu réguliers (ce sont seulement des mesures bornées). On obtient la stabilité lipschitz d'une primitive du multiplicateur associé à la contrainte sur l'état.

Mots-clés : comande optimale, contrainte sur l'état du second-ordre, analyse de stabilité, formulation alternative, condition d'optimalité suffisante du second-ordre, croissance quadratique uniforme, régularité forte. 


\section{Introduction}

This paper deals with stability analysis of nonlinear optimal control problems of an ordinary differential equation with a second-order state constraint. State constraints of second-order occur naturally in applications, see e.g. 7]. Stability and sensitivity analysis of solutions of optimal control problems is of high interest for the study of numerical methods, such as e.g. continuation algorithms, see 4, and to analyze the convergence of discretization schemes and obtain errors estimates, see e.g. [10].

For a class of general constrained optimization problems in Banach spaces, when the derivative of the constraint is "onto" and a second-order sufficient condition holds, Lipschitz stability of solutions and multipliers can be obtained by application of Robinson's strong regularity theory [27] to the first-order optimality system. For optimal control problems, this theory does not apply because of the well-known two-norm discrepancy (see 24]). Stability results for optimal control problems using variants of Robinson's strong regularity in order to deal with the two-norm approach have been obtained in [8], 17], 11] for control constraints, and 19 for mixed control-state constraints.

Lipschitz stability results for state constraints of first-order have been obtained by Malanowski [18] and Dontchev and Hager [9]. The difficulty of pure state constraints is the low regularity of multipliers, which are bounded Borel measures. For first-order state constraints, the multipliers are actually more regular (they are Lipschitz continuous functions, see Hager 14]). This additional regularity of solutions and multipliers is strongly used in the analysis in [18] and [9]. In those two papers, strong second-order sufficient conditions were used (that do not take into account the active constraints). The sufficient condition was recently weakened by Malanowski [21, 20.

For higher-order state constraints, the multipliers associated with the state constraints are only measures, and are not continuous w.r.t. the perturbation parameter (for the total variation norm). For this reason, the frameworks of [18, or 9] are not directly applicable. The only stability and sensitivity results known for state constraints of higher-order are based on the shooting approach, see Malanowski and Maurer [22] and [5]. Such results require strong assumptions on the structure of the contact set.

The main result of this paper is a stability result for regular second-order state constraints, with no assumption on the structure of the contact set. The control is assumed to be continuous and the strengthened Legendre-Clebsch condition to hold. We use a generalized implicit function theorem in metric spaces by Dontchev and Hager [9], applied to a system equivalent to the first-order optimality condition (the alternative formulation). This formulation involves alternative multipliers that are "integrals" of the original state constraint multipliers, and therefore are more regular. We obtain Lipschitz continuity of solutions and alternative multipliers in the $L^{2}$ norm, and Hölder continuity in the $L^{\infty}$ norm, under a weak second-order sufficient condition taking into account the active constraints.

The paper is organized as follows. In section 2, the problem, optimality conditions, assumptions, and the admissible class of perturbations are introduced. In section 3 , the second-order sufficient optimality condition is presented. In section 4, the main stability results for the nonlinear optimal control problem are given. Section 5 is devoted to stability 
analysis of linear-quadratic problems, that is used to prove the main theorem in section 6 Finally, conclusion and comments are given in section 7

\section{Preliminaries}

We consider the following optimal control problem

$$
\begin{aligned}
& \min _{(u, y) \in \mathcal{U} \times \mathcal{Y}} \int_{0}^{T} \ell(u(t), y(t)) \mathrm{d} t+\phi(y(T)) \\
& \dot{y}(t)=f(u(t), y(t)) \quad \text { for a.a. } t \in[0, T], \quad y(0)=y_{0} \\
& g(y(t)) \leq 0 \text { for all } t \in[0, T]
\end{aligned}
$$

with the control and state spaces $\mathcal{U}:=L^{\infty}\left(0, T ; \mathbb{R}^{m}\right)$ and $\mathcal{Y}:=W^{1, \infty}\left(0, T ; \mathbb{R}^{n}\right)$. The following assumptions are assumed to hold throughout the paper and will not be repeated in the various results of the paper.

(A0) The data $\ell: \mathbb{R}^{m} \times \mathbb{R}^{n} \rightarrow \mathbb{R}, \phi: \mathbb{R}^{n} \rightarrow \mathbb{R}\left(\right.$ resp. $f: \mathbb{R}^{m} \times \mathbb{R}^{n} \rightarrow \mathbb{R}^{n}, g: \mathbb{R}^{n} \rightarrow \mathbb{R}$ ) are $C^{2}$ (resp. $C^{3}, C^{4}$ ) mappings, with locally Lipschitz continuous second-order (resp. third-order, fourth order) derivatives, and $f$ is Lipschitz continuous.

(A1) The initial condition $y_{0} \in \mathbb{R}^{n}$ satisfies $g\left(y_{0}\right)<0$.

We consider in this paper state constraints of second-order. This means that the first-order time derivative $g^{(1)}: \mathbb{R}^{m} \times \mathbb{R}^{n} \rightarrow \mathbb{R}$ of the constraint, defined by

$$
g^{(1)}(u, y):=g_{y}(y) f(u, y)
$$

does not depend on the control variable $u$, i.e. $g_{u}^{(1)} \equiv 0$ (and hence, we write $g^{(1)}(y)=$ $g^{(1)}(u, y)$ ), and the second-order time derivative $g^{(2)}: \mathbb{R}^{m} \times \mathbb{R}^{n} \rightarrow \mathbb{R}$, defined by

$$
g^{(2)}(u, y):=g_{y}^{(1)}(y) f(u, y)
$$

depends explicitly on the control, i.e. $g_{u}^{(2)} \not \equiv 0$.

Remark 2.1. In this paper the state constraint is assumed to be scalar-valued for simplicity. The results are directly generalizable to several state constraints $g_{1}, \ldots, g_{r}$ of second-order (and even of higher-order 23, 15] $q_{i} \geq 2$ for $i=1, \ldots, r$, see Remark 2.2 further) under the assumption (see [23, 3]) that the gradients of the nearly active constraints $\nabla_{u} g_{i}^{\left(q_{i}\right)}(u, y)$ are uniformly linearly independent along the trajectory.

Notation We denote by subscripts Fréchet derivatives w.r.t. the variables $u$, $y$, i.e. $f_{y}(u, y)=D_{y} f(u, y), f_{y y}(u, y)=D_{y y}^{2} f(u, y)$, etc. The derivative with respect to the time is denoted by a dot, i.e. $\dot{y}=\frac{\mathrm{d} y}{\mathrm{~d} t}=y^{(1)}$. The set of row vectors of dimension $n$ is denoted 
by $\mathbb{R}^{n *}$. Adjoint or transpose operators are denoted by the symbol ${ }^{\top}$. The euclidean norm is denoted by $|\cdot|$. By $L^{r}(0, T)$ we denote the Lebesgue space of measurable functions such that $\|u\|_{r}:=\left(\int_{0}^{T}|u(t)|^{r} \mathrm{~d} t\right)^{1 / r}<\infty$ for $1 \leq r<\infty,\|u\|_{\infty}:=\operatorname{supess}_{[0, T]}|u(t)|<\infty$. The space $W^{s, r}(0, T)$ denotes the Sobolev space of functions having their $s$ first weak derivatives in $L^{r}(0, T)$, with the norm $\|u\|_{s, r}:=\sum_{j=0}^{s}\left\|u^{(j)}\right\|_{r}$. We denote by $H^{s}$ the space $W^{s, 2}$. The space of continuous functions over $[0, T]$ and its dual space, the space of bounded Borel measures, are denoted respectively by $C[0, T]$ and $\mathcal{M}[0, T]$. The set of nonnegative measures is denoted by $\mathcal{M}_{+}[0, T]$. The space of functions of bounded variation over $[0, T]$ is denoted by $B V[0, T]$, and the set of normalized BV functions vanishing at $T$ is denoted by $B V_{T}[0, T]$. Functions of bounded variation are w.l.o.g. assumed to be right-continuous. We identify the elements of $\mathcal{M}[0, T]$ with the distributional derivatives $\mathrm{d} \eta$ of functions $\eta$ in $B V_{T}[0, T]$. The support and the total variation of the measure $\mathrm{d} \eta \in \mathcal{M}[0, T]$ are denoted respectively by $\operatorname{supp}(\mathrm{d} \eta)$ and $|\mathrm{d} \eta|_{\mathcal{M}}$. The duality product over $\mathcal{M}[0, T] \times C[0, T]$ is denoted by $\langle\mathrm{d} \eta, x\rangle=\int_{0}^{T} x(t) \mathrm{d} \eta(t)$. We denote by $B_{X}(x, \rho)$ (resp. $B_{X}$ ) the open ball of the space $X$ with center $x$ and radius $\rho$ (resp. the open unit ball of the space $X$ ). We write $B_{r}$ for $B_{L^{r}}$, $r=2, \infty$.

We call a trajectory an element $(u, y) \in \mathcal{U} \times \mathcal{Y}$ satisfying the state equation 2.2. A trajectory satisfying the state constraint 2.3 is said to be feasible. The contact set of a feasible trajectory is defined by

$$
I(g(y)):=\{t \in[0, T]: g(y(t))=0\} .
$$

Under assumption (A0), the mapping $\mathcal{U} \rightarrow \mathcal{Y}, u \mapsto y_{u}$ where $y_{u}$ is the unique solution of the state equation (2.2), is well-defined. This leads us to the following abstract formulation of $(\mathcal{P})$ :

$$
\min _{u \in \mathcal{U}} J(u), \quad G(u) \in K,
$$

with the cost function $J(u):=\int_{0}^{T} \ell\left(u, y_{u}\right) \mathrm{d} t+\phi\left(y_{u}(T)\right)$, the constraint mapping $G(u):=$ $g\left(y_{u}\right)$, and the constraint cone $K:=C_{-}[0, T]$ is the cone of continuous functions taking nonpositive values over $[0, T]$. The polar cone to $K$, denoted by $K^{-}$, is the set of nonnegative measures $\mathcal{M}_{+}[0, T]$.

Finally, in all the paper the time argument $t \in[0, T]$ is often omitted when there is no ambiguity.

\subsection{Optimality conditions and Assumptions}

Let us first recall the well-known first-order necessary optimality condition of problem $(\mathcal{P})$.

The Hamiltonian $H: \mathbb{R}^{m} \times \mathbb{R}^{n} \times \mathbb{R}^{n *} \rightarrow \mathbb{R}$ is defined by

$$
H(u, y, p):=\ell(u, y)+p f(u, y) .
$$

RR $\mathrm{n}^{\circ} 0123456789$ 
We say that a feasible trajectory $(u, y)$ is a stationary point of $(\mathcal{P})$, if there exists $(p, \eta) \in$ $B V\left([0, T] ; \mathbb{R}^{n *}\right) \times B V_{T}[0, T]$ such that

$$
\begin{array}{rlr}
-\mathrm{d} p & =H_{y}(u, y, p) \mathrm{d} t+g_{y}(y) \mathrm{d} \eta, \quad p(T)=\phi_{y}(y(T)) \\
0 & =H_{u}(u(t), y(t), p(t)) \quad \text { a.e. on }[0, T] \\
\mathrm{d} \eta & \in N_{K}(g(y)) . &
\end{array}
$$

Here $N_{K}(g(y))$ denotes the normal cone to $K$ at point $g(y)$ (in the sense of convex analysis). If $g(y) \in K$, then $N_{K}(g(y))$ is the set of nonnegative measures in $\mathcal{M}_{+}[0, T]$ having their support included in the contact set 2.4), otherwise $N_{K}(g(y))$ is empty.

The Lagrangian $L: \mathcal{U} \times \mathcal{M}_{+}[0, T] \rightarrow \mathbb{R}$ of problem 2.5 is defined by

$$
L(u, \eta):=J(u)+\langle\mathrm{d} \eta, G(u)\rangle=J(u)+\int_{0}^{T} g\left(y_{u}(t)\right) \mathrm{d} \eta(t) .
$$

We may write the first-order optimality condition as follows: $\left(u, y=y_{u}\right)$ is a stationary point of $(\mathcal{P})$ iff there exists $\eta \in B V_{T}[0, T]$ such that

$$
D_{u} L(u, \eta)=0, \quad \mathrm{~d} \eta \in N_{K}(G(u)) .
$$

The costate $p$ is then obtained in function of $u, y=y_{u}$ and $\eta$ as the unique solution in $B V\left([0, T] ; \mathbb{R}^{n *}\right)$ of the costate equation 2.7$)$.

Robinson's constraint qualification [25, 26] for problem $(\mathcal{P})$ in abstract form $(2.5)$ is as follows:

$$
\exists \varepsilon>0, \quad \varepsilon B_{C[0, T]} \subset G(u)+D G(u) \mathcal{U}-K .
$$

This condition is equivalent to the existence of some $v \in \mathcal{U}$ such that

$$
D G(u) v<0 \quad \text { on } I(g(y)) .
$$

It is well-known that a local solution (weak minimum) of $(\mathcal{P})$ satisfying 2.12$)$ is a stationary point of $(\mathcal{P})$.

Alternative formulation For the stability analysis, it will be convenient to write the optimality condition using alternative multipliers $\eta^{2}$ and $p^{2}$, uniquely related to $(p, \eta)$ in the following way:

$$
\begin{aligned}
\eta^{1}(t) & :=-\int_{] t, T]} \mathrm{d} \eta(s)=-\eta(t), \quad \eta^{2}(t):=\int_{t}^{T} \eta^{1}(s) \mathrm{d} s, \\
p^{2}(t) & :=p(t)-\eta^{1}(t) g_{y}(y(t))-\eta^{2}(t) g_{y}^{(1)}(y(t)), \quad t \in[0, T] .
\end{aligned}
$$

We see that $\eta^{2}$ belongs to the set $B V_{T}^{2}[0, T]$, defined by

$$
B V_{T}^{2}[0, T]:=\left\{\xi \in W^{1, \infty}(0, T): \xi(T)=0, \dot{\xi} \in B V_{T}[0, T]\right\} .
$$


Define the alternative Hamiltonian $\tilde{H}: \mathbb{R}^{m} \times \mathbb{R}^{n} \times \mathbb{R}^{n *} \times \mathbb{R} \rightarrow \mathbb{R}$ by

$$
\tilde{H}\left(u, y, p^{2}, \eta^{2}\right):=H\left(u, y, p^{2}\right)+\eta^{2} g^{(2)}(u, y),
$$

where $H$ is the classical Hamiltonian (2.6). Using these alternative multipliers, it is not difficult to see by a direct calculation (see 23] or [3, Lemma 3.4]) that a feasible trajectory $(u, y)$ is a stationary point of $(\mathcal{P})$ iff there exists $\left(p^{2}, \eta^{2}\right) \in W^{1, \infty}\left(0, T ; \mathbb{R}^{n *}\right) \times B V_{T}^{2}[0, T]$ such that

$$
\begin{array}{rlrl}
-\dot{p}^{2} & =\tilde{H}_{y}\left(u, y, p^{2}, \eta^{2}\right), & & p^{2}(T)=\phi_{y}(y(T)) \\
0 & =\tilde{H}_{u}\left(u, y, p^{2}, \eta^{2}\right) & \text { a.e. on }[0, T] \\
\mathrm{d} \dot{\eta}^{2} & \in N_{K}(g(y)) . &
\end{array}
$$

The definition of these multipliers $p^{2}, \eta^{2}$ is inspired by the ones used in the alternative formulation for the shooting algorithm, see [23, 15, 22, 5], though $p^{2}, \eta^{2}$ are continuous over $[0, T]$ while the ones in the shooting algorithm have jumps.

Remark 2.2. The results of this paper have a natural generalization to a state constraint of higher-order $q>2$, considering in the analysis alternative multipliers $\left(\eta^{q}, p^{q}\right)$ of order $q$ defined below and the resulting alternative formulation of optimality condition of order $q$. These alternative multipliers of order $q, \eta^{q} \in B V_{T}^{q}[0, T]$ with

$$
B V_{T}^{q}[0, T]:=\left\{\xi \in W^{q-1, \infty}(0, T): \xi^{(j)}(T)=0 \forall j=0, \ldots, q-2, \xi^{(q-1)} \in B V_{T}[0, T]\right\}
$$

and $p^{q} \in W^{1, \infty}\left(0, T ; \mathbb{R}^{n *}\right)$, are defined by

$$
\begin{aligned}
\eta^{1}(t) & :=-\int_{] t, T]} \mathrm{d} \eta(s), \quad \eta^{j}(t):=\int_{t}^{T} \eta^{j-1}(s) \mathrm{d} s, \quad j=2, \ldots, q, \\
p^{q}(t) & :=p(t)-\sum_{j=1}^{q} \eta^{j}(t) g_{y}^{(j-1)}(y(t)) .
\end{aligned}
$$

Assumptions Let $(\bar{u}, \bar{y})$ be a local solution of $(\mathcal{P})$. We denote by $\Omega:=I(g(\bar{y}))$ the contact set of the trajectory $(\bar{u}, \bar{y})$, and for a small $\sigma>0$, let $\Omega_{\sigma}$ denote a neighborhood of the contact set

$$
\Omega_{\sigma}:=\{t \in[0, T]: \operatorname{dist}\{t, \Omega\}<\sigma\} .
$$

We assume that $(\bar{u}, \bar{y})$ satisfies the assumption below:

(A2) The state constraint is a regular second-order state constraint, i.e. $g_{u}^{(1)} \equiv 0$ and

$$
\exists \beta, \sigma>0, \quad\left|g_{u}^{(2)}(\bar{u}(t), \bar{y}(t))\right| \geq \beta, \quad \text { for a.a. } t \in \Omega_{\sigma} .
$$

Given $v \in L^{r}\left(0, T ; \mathbb{R}^{m}\right), 1 \leq r \leq \infty$, we denote by $z_{v}$ the unique solution in $W^{1, r}\left(0, T ; \mathbb{R}^{n}\right)$ of the linearized state equation

$$
\dot{z}_{v}(t)=f_{y}(\bar{u}(t), \bar{y}(t)) z_{v}(t)+f_{u}(\bar{u}(t), \bar{y}(t)) v(t) \quad \text { a.e. on }[0, T], \quad z_{v}(0)=0 .
$$

Note that the derivative of the constraint mapping is given by $D G(\bar{u}) v=g_{y}(\bar{y}) z_{v}$. 
Lemma 2.3. Let $(\bar{u}, \bar{y})$ be a feasible trajectory of $(\mathcal{P})$ satisfying (A2). Then for all $r \in$ $[1,+\infty]$ and all $\varepsilon \in(0, \sigma)$, with the $\sigma$ of $(2.21)$, so small that

$$
\Omega_{\varepsilon} \subset[a, T], \quad \text { for some } a>0,
$$

the linear mapping

$$
L^{r}\left(0, T ; \mathbb{R}^{m}\right) \rightarrow W^{2, r}\left(\Omega_{\varepsilon}\right),\left.\quad v \mapsto\left(g_{y}(\bar{y}(\cdot)) z_{v}(\cdot)\right)\right|_{\Omega_{\varepsilon}},
$$

where $\left.\right|_{\Omega_{\varepsilon}}$ denotes the restriction to the set $\Omega_{\varepsilon}$, is onto, and therefore has a bounded right inverse by the open mapping theorem.

Proof. We only recall the main ideas of the proof, given in [3, Lemma 2.2]. We have that

$$
\begin{aligned}
\frac{\mathrm{d}}{\mathrm{d} t}\left\{g_{y}(\bar{y}(t)) z_{v}(t)\right\} & =g_{y}^{(1)}(\bar{y}(t)) z_{v}(t), \\
\frac{\mathrm{d}^{2}}{\mathrm{~d} t^{2}}\left\{g_{y}(\bar{y}(t)) z_{v}(t)\right\} & =g_{y}^{(2)}(\bar{u}(t), \bar{y}(t)) z_{v}(t)+g_{u}^{(2)}(\bar{u}(t), \bar{y}(t)) v(t) .
\end{aligned}
$$

Since by (A1) and hypothesis 2.21$), g_{u}^{(2)}(\bar{u}(t), \bar{y}(t))$ is non singular on a left neighborhood of $\Omega_{\varepsilon}$, the result follows from Gronwall's Lemma.

By the above lemma, assumption (A2) (together with (A1)) implies that $(\bar{u}, \bar{y})$ satisfies Robinson's constraint qualification $(2.12$, and hence $(\bar{u}, \bar{y})$ is a stationary point of $(\mathcal{P})$, with multipliers $(\bar{p}, \bar{\eta})$. Moreover, Lemma 2.3 implies that the multipliers $(\bar{p}, \bar{\eta})$ associated with $(\bar{u}, \bar{y})$ are unique. We assume in addition that

(A3) $\bar{u}$ is continuous on $[0, T]$ and the strengthened Legendre-Clebsch condition holds:

$$
\exists \alpha>0, v^{\top} H_{u u}(\bar{u}(t), \bar{y}(t), \bar{p}(t)) v \geq \alpha|v|^{2}, \text { for all } t \in[0, T] \text { and all } v \in \mathbb{R}^{m} .
$$

Remark 2.4. A stronger assumption than (2.25), which implies the continuity of $\bar{u}$ (see 3 , Prop. 3.1]), is the uniform strong convexity of the Hamiltonian:

$$
\exists \alpha>0, v^{\top} H_{u u}(\hat{u}, \bar{y}(t), \bar{p}(t)) v \geq \alpha|v|^{2}, \quad \text { for all } t \in[0, T] \text { and all } \hat{u}, v \in \mathbb{R}^{m} .
$$

Denote by $\bar{p}^{2}$ and $\bar{\eta}^{2}$ the alternative multipliers related to $\bar{p}$ and $\bar{\eta}$ by (2.13)-(2.14). Assumption 2.25 can be rewritten, using the alternative multipliers $\bar{p}^{2}$ and $\bar{\eta}^{2}$ instead of $\bar{p}$ and $\bar{\eta}$ and the alternative Hamiltonian (2.16), by:

$$
\exists \alpha>0, v^{\top} \tilde{H}_{u u}\left(\bar{u}(t), \bar{y}(t), \bar{p}^{2}(t), \bar{\eta}^{2}(t)\right) v \geq \alpha|v|^{2}, \text { for all } t \in[0, T] \text { and all } v \in \mathbb{R}^{m} \text {. }
$$

Lemma 2.5. Let $(\bar{u}, \bar{y})$ be a stationary point of $(\mathcal{P})$ satisfying (A2)-(A3). Then $\bar{u} \in$ $W^{1, \infty}\left(0, T ; \mathbb{R}^{m}\right)$. 
Proof. By (A3), implying (2.26), and the implicit function theorem applied to relation (2.18), there exists a $C^{1}$ function $\Upsilon$ such that $\bar{u}(t)=\Upsilon\left(\bar{y}(t), \bar{p}^{2}(t), \bar{\eta}^{2}(t)\right)$. Since $\bar{y}, \bar{p}^{2}, \bar{\eta}^{2} \in W^{1, \infty}$, it follows from the chain rule that $\bar{u} \in W^{1, \infty}$.

Remark 2.6. More precisely, we have that under the assumptions of Lemma 2.5. $\bar{u} \in$ $B V^{2}\left([0, T] ; \mathbb{R}^{m}\right)$, where $B V^{2}[0, T]:=\left\{u \in W^{1, \infty}(0, T): \dot{u} \in B V[0, T]\right\}$. Indeed, differentiation of 2.18 w.r.t. time shows that (omitting arguments $\left(\bar{u}, \bar{y}, \bar{p}^{2}, \bar{\eta}^{2}\right)$ )

$$
0=\tilde{H}_{u u} \dot{\bar{u}}+\tilde{H}_{u y} f-\tilde{H}_{y} f_{u}+\dot{\bar{\eta}}^{2} g_{u}^{(2)} \text {. }
$$

Since $\dot{\bar{\eta}}^{2}=\bar{\eta} \in B V_{T}[0, T]$ and $\tilde{H}_{u u}$ is uniformly invertible by $(2.26)$, we obtain the result.

\subsection{Perturbed optimal control problem}

We consider perturbed problems in the following form:

$$
\begin{aligned}
& \min _{(u, y) \in \mathcal{U} \times \mathcal{Y}} \int_{0}^{T} \ell^{\mu}(u(t), y(t)) \mathrm{d} t+\phi^{\mu}(y(T)) \\
& \dot{y}(t)=f^{\mu}(u(t), y(t)) \quad \text { a.e. on }[0, T], \quad y(0)=y_{0}^{\mu} \\
& g^{\mu}(y(t)) \leq 0 \text { for all } t \in[0, T] .
\end{aligned}
$$

Here $\mu$ is the perturbation parameter, living in an open subset $M_{0}$ of a Banach space $M$.

Definition 2.7. We say that $\left(\mathcal{P}^{\mu}\right)$ is a stable extension of $(\mathcal{P})$, if:

(i) There exists $\bar{\mu} \in M_{0}$ such that $\left(\mathcal{P}^{\bar{\mu}}\right) \equiv(\mathcal{P})$;

(ii) The mappings $\mathbb{R}^{m} \times \mathbb{R}^{n} \times M_{0} \rightarrow \mathbb{R},(u, y, \mu) \mapsto \ell^{\mu}(u, y) ; \mathbb{R}^{n} \times M_{0} \rightarrow \mathbb{R},(y, \mu) \mapsto \phi^{\mu}(y)$; $M_{0} \rightarrow \mathbb{R}^{n}, \mu \mapsto y_{0}^{\mu}\left(\right.$ resp. $\mathbb{R}^{m} \times \mathbb{R}^{n} \times M_{0} \rightarrow \mathbb{R}^{n},(u, y, \mu) \mapsto f^{\mu}(u, y) ; \mathbb{R}^{n} \times M_{0} \rightarrow \mathbb{R}$, $\left.(y, \mu) \mapsto g^{\mu}(y)\right)$ are of class $C^{2}$ (resp. $C^{3}, C^{4}$ ), with locally Lipschitz continuous secondorder (resp. third-order, fourth order) derivatives, uniformly w.r.t. $\mu \in M_{0}$;

(iii) $f^{\mu}$ is uniformly Lipschitz continuous over $\mathbb{R}^{m} \times \mathbb{R}^{n}$ for all $\mu \in M_{0}$;

(iv) the state constraint is not of first-order, i.e. $\left(g^{\mu}\right)_{u}^{(1)}(u, y) \equiv 0$ for all $(u, y, \mu) \in \mathbb{R}^{m} \times$ $\mathbb{R}^{n} \times M_{0}$.

Given a stable extension $\left(\mathcal{P}^{\mu}\right)$ and $(u, \mu) \in \mathcal{U} \times M_{0}$, we denote by $y_{u}^{\mu}$ the unique solution in $\mathcal{Y}$ of the state equation (2.28), and we have the abstract formulation of $\left(\mathcal{P}^{\mu}\right)$

$$
\min _{u \in \mathcal{U}} J^{\mu}(u), \quad G^{\mu}(u) \in K,
$$

with $J^{\mu}(u):=\int_{0}^{T} \ell^{\mu}\left(u, y_{u}^{\mu}\right) \mathrm{d} t+\phi^{\mu}\left(y_{u}^{\mu}(T)\right)$ and $G^{\mu}(u):=g^{\mu}\left(y_{u}^{\mu}\right)$. When we refer to the data of the reference problem $(\mathcal{P})$, we often omit the superscript $\bar{\mu}$.

\section{Second-order sufficient optimality condition}

Let $(\bar{u}, \bar{y})$ be a stationary point of $(\mathcal{P})$, with multipliers $(\bar{p}, \bar{\eta})$. Let $\mathcal{V}:=L^{2}\left(0, T ; \mathbb{R}^{m}\right)$. The quadratic form involved in the second-order optimality conditions, defined over $\mathcal{V}$, is as 
follows:

$$
\begin{aligned}
\mathcal{Q}(v):= & \int_{0}^{T} D_{(u, y)^{2}}^{2} H(\bar{u}, \bar{y}, \bar{p})\left(v, z_{v}\right)^{2} \mathrm{~d} t+\phi_{y y}(\bar{y}(T))\left(z_{v}(T), z_{v}(T)\right) \\
& +\int_{0}^{T} g_{y y}(\bar{y})\left(z_{v}, z_{v}\right) \mathrm{d} \bar{\eta} .
\end{aligned}
$$

Recall that $z_{v}$ is the solution of the linearized state equation $(2.22)$. Here the notation $D_{(u, y)^{2}}^{2} H(\bar{u}, \bar{y}, \bar{p})\left(v, z_{v}\right)^{2}$ stands for $D_{(u, y)(u, y)}^{2} H(\bar{u}, \bar{y}, \bar{p})\left(\left(v, z_{v}\right),\left(v, z_{v}\right)\right)$. The critical cone $\mathcal{C}(\bar{u})$ is the set of $v \in \mathcal{V}$ satisfying

$$
\begin{array}{ll}
g_{y}(\bar{y}(t)) z_{v}(t)=0 & \text { on } \operatorname{supp}(\mathrm{d} \bar{\eta}), \\
g_{y}(\bar{y}(t)) z_{v}(t) \leq 0 & \text { on } I(g(\bar{y})) \backslash \operatorname{supp}(\mathrm{d} \bar{\eta}) .
\end{array}
$$

A sufficient second-order optimality condition for $(\mathcal{P})$ is, see [2, Th. 18] for scalar-valued control and constraint and [3, Th. 6.1] for vector-valued ones:

$$
\mathcal{Q}(v)>0, \quad \text { for all } v \in \mathcal{C}(\bar{u}) \backslash\{0\} .
$$

When the strengthened Legendre-Clebsch condition (2.25) holds, (3.4) implies that $(\bar{u}, \bar{y})$ is a local solution of $(\mathcal{P})$ satisfying the second-order growth condition:

$$
\exists c, \rho>0, \quad J(u) \geq J(\bar{u})+c\|u-\bar{u}\|_{2}^{2}, \quad \text { for all } u \in \mathcal{U}: G(u) \in K,\|u-\bar{u}\|_{\infty}<\rho .
$$

This condition involves two norms, $L^{2}$ for the growth condition and $L^{\infty}$ for the neighborhood.

We will use, in the stability analysis, a natural strengthening of the sufficient condition (3.4), omitting the inequality constraint (3.3) in the critical cone. So let the extended critical cone $\hat{\mathcal{C}}(\bar{u})$ be defined as the set of $v \in \mathcal{V}$ satisfying $(3.2)$ (and hence, $\mathcal{C}(\bar{u}) \subset \hat{\mathcal{C}}(\bar{u})$ ). The strong second-order sufficient condition used in the stability analysis is as follows:

$$
\mathcal{Q}(v)>0, \quad \text { for all } v \in \hat{\mathcal{C}}(\bar{u}) \backslash\{0\} .
$$

Although we call the above condition the strong second-order sufficient condition (in comparison with (3.4)), it takes into account the active constraints so it is weaker than the second-order sufficient condition used in [9] that assumes the strict positivity of $\mathcal{Q}$ over the whole space $\mathcal{V} \backslash\{0\}$.

The strengthened Legendre-Clebsch condition (2.25) implies (see [6, Prop. 3.76(i)]) that the quadratic form $\mathcal{Q}$ is a Legendre form (see [16]), i.e. a weakly lower semi-continuous (weakly l.s.c.) quadratic form with the property that if a sequence $v_{n}$ weakly converges to $v$ in $L^{2}\left(v_{n} \rightarrow v\right)$ and if $Q\left(v_{n}\right) \rightarrow Q(v)$, then $v_{n} \rightarrow v$ strongly.

Lemma 3.1. Let $(\bar{u}, \bar{y})$ be a stationary point of $(\mathcal{P})$. An equivalent expression for the quadratic form $\mathcal{Q}$ defined by (3.1), using the alternative multipliers $\left(\bar{p}^{2}, \bar{\eta}^{2}\right)$ given by (2.13)(2.14) instead of $(\bar{p}, \bar{\eta})$ and the alternative Hamiltonian (2.16), is:

$$
\mathcal{Q}(v)=\int_{0}^{T} D_{(u, y)^{2}}^{2} \tilde{H}\left(\bar{u}, \bar{y}, \bar{p}^{2}, \bar{\eta}^{2}\right)\left(v, z_{v}\right)^{2} \mathrm{~d} t+\phi_{y y}(\bar{y}(T))\left(z_{v}(T), z_{v}(T)\right) .
$$


Proof. Let $v \in \mathcal{V}$. Denote by $\tilde{\mathcal{Q}}(v)$ the right-hand side of $(3.7)$ and set $\Delta:=\tilde{\mathcal{Q}}(v)-\mathcal{Q}(v)$. In view of the relations $2.13-2.14$ between $\left(\bar{p}^{2}, \bar{\eta}^{2}\right)$ and $(\bar{p}, \bar{\eta})$, we have

$$
\begin{aligned}
\Delta= & \int_{0}^{T}\left(\bar{p}^{2}-\bar{p}\right) D^{2} f(\bar{u}, \bar{y})\left(v, z_{v}\right)^{2} \mathrm{~d} t+\int_{0}^{T} D^{2} g^{(2)}(\bar{u}, \bar{y})\left(v, z_{v}\right)^{2} \bar{\eta}^{2} \mathrm{~d} t \\
& -\int_{0}^{T} g_{y y}(\bar{y})\left(z_{v}, z_{v}\right) \mathrm{d} \bar{\eta} \\
= & -\int_{0}^{T} \bar{\eta}^{1} g_{y}(\bar{y}) D^{2} f(\bar{u}, \bar{y})\left(v, z_{v}\right)^{2} \mathrm{~d} t-\int_{0}^{T} \bar{\eta}^{2} g_{y}^{(1)}(\bar{y}) D^{2} f(\bar{u}, \bar{y})\left(v, z_{v}\right)^{2} \mathrm{~d} t \\
& +\int_{0}^{T} D^{2} g^{(2)}(\bar{u}, \bar{y})\left(v, z_{v}\right)^{2} \bar{\eta}^{2} \mathrm{~d} t-\int_{0}^{T} g_{y y}(\bar{y})\left(z_{v}, z_{v}\right) \mathrm{d} \bar{\eta} .
\end{aligned}
$$

The integration by parts formula in BV [12, p.154] shows that (the calculus is analogous to Lemma 3.6 in [5])

$$
\begin{aligned}
\int_{0}^{T} g_{y y}(\bar{y})\left(z_{v}, z_{v}\right) \mathrm{d} \bar{\eta} & =\int_{0}^{T} \frac{\mathrm{d}}{\mathrm{d} t}\left\{g_{y y}(\bar{y})\left(z_{v}, z_{v}\right)\right\} \bar{\eta}^{1} \mathrm{~d} t+\left[g_{y y}(\bar{y})\left(z_{v}, z_{v}\right) \bar{\eta}^{1}\right]_{0}^{T} \\
& =\int_{0}^{T}\left\{g_{y y y}(\bar{y})\left(f, z_{v}, z_{v}\right)+2 g_{y y}(\bar{y})\left(D f(\bar{u}, \bar{y})\left(v, z_{v}\right), z_{v}\right)\right\} \bar{\eta}^{1} \mathrm{~d} t \\
& =\int_{0}^{T} g_{y y}^{(1)}(\bar{y})\left(z_{v}, z_{v}\right) \bar{\eta}^{1} \mathrm{~d} t-\int_{0}^{T} g_{y}(\bar{y}) D^{2} f(\bar{u}, \bar{y})\left(v, z_{v}\right)^{2} \bar{\eta}^{1} \mathrm{~d} t
\end{aligned}
$$

Similarly, we obtain that

$$
\int_{0}^{T} g_{y y}^{(1)}(\bar{y})\left(z_{v}, z_{v}\right) \bar{\eta}^{1} \mathrm{~d} t=\int_{0}^{T} D^{2} g^{(2)}(\bar{u}, \bar{y})\left(v, z_{v}\right)^{2} \bar{\eta}^{2} \mathrm{~d} t-\int_{0}^{T} g_{y}^{(1)}(\bar{y}) D^{2} f(\bar{u}, \bar{y})\left(v, z_{v}\right)^{2} \bar{\eta}^{2} \mathrm{~d} t .
$$

Summing the two above equalities, we obtain that $\Delta=0$, which completes the proof.

\section{Stability analysis for the nonlinear problem}

According to Def. 5.16 in [6], adapted to our optimal control framework, we consider the following definition of uniform second-order growth condition.

Definition 4.1. Let $(\bar{u}, \bar{y})$ be a stationary point of $(\mathcal{P})$. We say that the uniform second-order (or quadratic) growth condition holds, if for all stable extensions $\left(\mathcal{P}^{\mu}\right)$ of $(\mathcal{P})$, there exists $c, \rho>0$ and a neighborhood $\mathcal{N}$ of $\bar{\mu}$, such that for any stationary point $\left(u^{\mu}, y^{\mu}\right)$ of $\left(\mathcal{P}^{\mu}\right)$ with $\mu \in \mathcal{N}$ and $\left\|u^{\mu}-\bar{u}\right\|_{\infty}<\rho$,

$$
J^{\mu}(u) \geq J^{\mu}\left(u^{\mu}\right)+c\left\|u-u^{\mu}\right\|_{2}^{2}, \quad \text { for all } u \in \mathcal{U}: G^{\mu}(u) \in K,\|u-\bar{u}\|_{\infty}<\rho .
$$

The next proposition (proved in subsection 4.2 shows that the strong second-order sufficient condition (3.6) implies the uniform second-order growth condition. Therefore, if a 
stationary point for the perturbed problem $\left(\mathcal{P}^{\mu}\right)$ exists, then the latter is locally unique in a $L^{\infty}$-neighborhood of $\bar{u}$, and is a local solution of $\left(\mathcal{P}^{\mu}\right)$.

Proposition 4.2. Let $(\bar{u}, \bar{y})$ be a stationary point of $(\mathcal{P})$ satisfying (A2)-(A3) and the strong second-order sufficient condition (3.6). Then the uniform second-order growth condition holds.

The difficult part in the stability analysis here is to prove the existence of a stationary point for the perturbed problem. For some general optimization problems, Robinson's constraint qualification 2.12 and the uniform quadratic growth condition imply, for a certain class of perturbations, the existence of a stationary point for the perturbed problem, see Bonnans and Shapiro [6, Th. 5.17]. The proof uses Ekeland's variational principle [13. However, this result does not apply to our nonlinear optimal control problem, due to the two-norms discrepancy, but it does for a linear-quadratic problem (see the proof of Th. 5.4.) For the general nonlinear problem, in order to obtain the existence of a stationary point for the perturbed problem, we need to use a variant of Robinson's strong regularity theory [27.

The main result of the paper is the next theorem (proved in section 6).

Theorem 4.3. Let $(\bar{u}, \bar{y})$ be a local solution of $(\mathcal{P})$, satisfying (A2)-(A3) and the strong second-order sufficient condition (3.6). Then for all stable extensions $\left(\mathcal{P}^{\mu}\right)$ of $(\mathcal{P})$, there exist $c, \rho, \kappa, \tilde{\kappa}>0$ and a neighborhood $\mathcal{N}$ of $\bar{\mu}$, such that for all $\mu \in \mathcal{N},\left(\mathcal{P}^{\mu}\right)$ has a unique stationary point $\left(u^{\mu}, y^{\mu}\right)$ with $\left\|u^{\mu}-\bar{u}\right\|_{\infty}<\rho$ and unique associated alternative multipliers $\left(p^{2, \mu}, \eta^{2, \mu}\right)$, and for all $\mu, \mu^{\prime} \in \mathcal{N}$,

$$
\begin{aligned}
&\left\|u^{\mu}-u^{\mu^{\prime}}\right\|_{2},\left\|y^{\mu}-y^{\mu^{\prime}}\right\|_{1,2},\left\|p^{2, \mu}-p^{2, \mu^{\prime}}\right\|_{1,2},\left\|\eta^{2, \mu}-\eta^{2, \mu^{\prime}}\right\|_{2} \leq \kappa\left\|\mu-\mu^{\prime}\right\|, \\
&\left\|u^{\mu}-u^{\mu^{\prime}}\right\|_{\infty},\left\|y^{\mu}-y^{\mu^{\prime}}\right\|_{1, \infty},\left\|p^{2, \mu}-p^{2, \mu^{\prime}}\right\|_{1, \infty},\left\|\eta^{2, \mu}-\eta^{2, \mu^{\prime}}\right\|_{\infty} \leq \tilde{\kappa}\left\|\mu-\mu^{\prime}\right\|^{2 / 3} .
\end{aligned}
$$

Moreover, $\left(u^{\mu}, y^{\mu}\right)$ is a local solution of $\left(\mathcal{P}^{\mu}\right)$ satisfying the uniform quadratic growth condition 4.1.

The above theorem is obtained by application of a generalized implicit function theorem by Dontchev and Hager [9] (Th. 4.8 of this paper) to the alternative formulation (2.17)2.19 in suitable functional spaces described in subsection 4.3. In order to show that the main assumption of this theorem is satisfied (assumption (iv)), we have to show that a perturbed linear-quadratic optimal control problem has a unique solution which is Lipschitz continuous w.r.t. the parameter. For this, we will use Prop. 4.2 (or more precisely, its analogous statement adapted to linear-quadratic problems.) Before giving the proof of Prop. 4.2, we first need to study the stability of multipliers (Prop. 4.4).

\subsection{Stability of multipliers}

The next result shows that under the constraint qualification (A2), the stability of multipliers could be deduced from the stability of solutions. Given $r \in[1,+\infty]$, we denote by $\|\cdot\|_{2, r *}$ 
the norm of the dual space to $W^{2, r}(0, T)$, i.e., for $\mathrm{d} \eta \in \mathcal{M}[0, T]$ we have

$$
\|\mathrm{d} \eta\|_{2, r *}:=\sup \left\{\frac{\left|\int_{0}^{T} \Phi(t) \mathrm{d} \eta(t)\right|}{\|\Phi\|_{2, r}}, \Phi \in W^{2, r}(0, T), \Phi \not \equiv 0\right\} .
$$

Proposition 4.4. Let $(\bar{u}, \bar{y})$ be a stationary point of $(\mathcal{P})$ satisfying (A2). Then for every stable extension $\left(\mathcal{P}^{\mu}\right)$ of $(\mathcal{P})$, there exists $\nu>0$ such that for every stationary point $(u, y)$ of $\left(\mathcal{P}^{\mu}\right)$, with (unique) associated multipliers $(p, \eta)$ and alternative multipliers $\left(p^{2}, \eta^{2}\right)$ given by 2.13-2.14), the following hold:

(i) If $\|\mu-\bar{\mu}\|,\|u-\bar{u}\|_{\infty}<\nu$, then $\mathrm{d} \eta$ is uniformly bounded in $\mathcal{M}[0, T]$;

(ii) There exists $\kappa>0$ such that, for all $\|\mu-\bar{\mu}\|,\|u-\bar{u}\|_{\infty}<\nu$,

$$
\|\mathrm{d} \eta-\mathrm{d} \bar{\eta}\|_{2,1 *},\left\|\eta^{2}-\bar{\eta}^{2}\right\|_{\infty} \leq \kappa\left(\|u-\bar{u}\|_{\infty}+\|\mu-\bar{\mu}\|\right)
$$

Moreover, when $\|\mu-\bar{\mu}\|,\|u-\bar{u}\|_{\infty} \rightarrow 0$ :

(iii) $\mathrm{d} \eta$ weakly- * converges to $\mathrm{d} \bar{\eta}(\mathrm{d} \eta \stackrel{*}{\rightarrow} \mathrm{d} \bar{\eta})$ in $\mathcal{M}[0, T]$;

(iv) $\eta^{1} \rightarrow \bar{\eta}^{1}$ in $L^{1}$;

(v) $p^{2}$ and $\eta^{2}$ converges uniformly to $\bar{p}^{2}$ and $\bar{\eta}^{2}$, respectively.

The proof of the above proposition uses the lemma below.

Lemma 4.5. For all $1 \leq r<\infty$, with $r^{\prime}:=r /(r-1)\left(1^{\prime}=\infty\right)$, there exists a positive constant $C$ such that

$$
\|\xi\|_{r^{\prime}} \leq C\|\mathrm{~d} \dot{\xi}\|_{2, r *} \quad \text { for all } \xi \in B V_{T}^{2}[0, T]
$$

Proof. Let $\varphi \in L^{r}(0, T)$. Set $\Phi^{1}(t):=\int_{0}^{t} \varphi(s) \mathrm{d} s$ and $\Phi(t):=\int_{0}^{t} \Phi^{1}(s) \mathrm{d} s$. Then $\Phi \in$ $W^{2, r}(0, T)$, and $\|\Phi\|_{2, r} \leq C\|\varphi\|_{r}$, with $C=1+T / \sqrt[r]{r}+(T / \sqrt[r]{r})^{2}$. For all $\xi \in B V_{T}^{2}[0, T]$, using that $\xi(T)=\dot{\xi}(T)=0$, the integration by parts formula in BV [12, p.154] implies that

$$
\int_{0}^{T} \varphi(t) \xi(t) \mathrm{d} t=-\int_{0}^{T} \Phi^{1}(t) \dot{\xi}(t) \mathrm{d} t=\int_{0}^{T} \Phi(t) \mathrm{d} \dot{\xi}(t) .
$$

Therefore,

$$
\|\xi\|_{r^{\prime}}=\sup _{\varphi \in L^{r}, \varphi \neq \equiv 0} \frac{\left|\int_{0}^{T} \varphi(t) \xi(t) \mathrm{d} t\right|}{\|\varphi\|_{r}} \leq C \sup _{\Phi \in W^{2, r}, \Phi \neq 0} \frac{\left|\int_{0}^{T} \Phi(t) \mathrm{d} \dot{\xi}(t)\right|}{\|\Phi\|_{2, r}}
$$

which gives the result.

Proof of Prop. 4.4. Let $\left(\mathcal{P}^{\mu}\right)$ be a stable extension of $(\mathcal{P})$. Note first that for $\|\mu-\bar{\mu}\|$ and $\|u-\bar{u}\|_{\infty}$ small enough, assumptions (A1) and (A2) hold for $\left(\mathcal{P}^{\mu}\right)$. This implies the uniqueness of the multipliers $(p, \eta)$ associated with a stationary point $(u, y)$ of $\left(\mathcal{P}^{\mu}\right)$. Since $(\bar{u}, \bar{y})$ satisfies Robinson's constraint qualification (2.12), point (i) follows from [6, Prop. 4.43].

$\mathrm{RR} \quad \mathrm{n}^{\circ} 0123456789$ 
Let us show (ii). Since $\left(u, y=y_{u}^{\mu}\right)$ is a stationary point of $\left(\mathcal{P}^{\mu}\right)$, we have that

$$
D J^{\mu}(u)+D G^{\mu}(u)^{\top} \mathrm{d} \eta=0, \quad \mathrm{~d} \eta \in N_{K}\left(G^{\mu}(u)\right) .
$$

It follows that $D G(\bar{u})^{\top}(\mathrm{d} \bar{\eta}-\mathrm{d} \eta)=D J^{\mu}(u)-D J(\bar{u})+\left(D G^{\mu}(u)-D G(\bar{u})\right)^{\top} \mathrm{d} \eta$, and hence, for all $v \in L^{1}(0, T)$,

$$
\langle\mathrm{d} \bar{\eta}-\mathrm{d} \eta, D G(\bar{u}) v\rangle=\left(D J^{\mu}(u)-D J(\bar{u})\right) v+\left\langle\mathrm{d} \eta,\left(D G^{\mu}(u)-D G(\bar{u})\right) v\right\rangle .
$$

Fix $\varepsilon \in(0, \sigma)$ with the $\sigma$ of $(2.21)$ satisfying (2.23). By Lemma 2.3, the linear mapping defined in 2.24 for $r=1$ is onto. By the open mapping theorem, there exists a constant $C_{1}>0$ such that for all $\Phi \in W^{2,1}(0, T)$, there exists $v \in L^{1}(0, T)$ such that $D G(\bar{u}) v=$ $\Phi$ on $\Omega_{\varepsilon}$ and $\|v\|_{1} \leq C_{1}\|\Phi\|_{2,1}$. For $\|\mu-\bar{\mu}\|,\|u-\bar{u}\|_{\infty}$ small enough, the contact set $I\left(g^{\mu}(y)\right)$, and hence the support of the measure $\mathrm{d} \eta$, are included in the set $\Omega_{\varepsilon}$. Therefore, $\langle\mathrm{d} \eta-\mathrm{d} \bar{\eta}, D G(\bar{u}) v\rangle=\langle\mathrm{d} \eta-\mathrm{d} \bar{\eta}, \Phi\rangle$. Consequently, by (4.5),

$$
|\langle\mathrm{d} \eta-\mathrm{d} \bar{\eta}, \Phi\rangle| \leq\left|\left(D J^{\mu}(u)-D J(\bar{u})\right) v\right|+|\mathrm{d} \eta|_{\mathcal{M}}\left\|\left(D G^{\mu}(u)-D G(\bar{u})\right) v\right\|_{\infty} .
$$

By point (i), $|\mathrm{d} \eta|_{\mathcal{M}}$ is uniformly bounded, and it is not difficult to check that

$$
\left|\left(D J^{\mu}(u)-D J(\bar{u})\right) v\right|,\left\|\left(D G^{\mu}(u)-D G(\bar{u})\right) v\right\|_{\infty} \leq C\left(\|u-\bar{u}\|_{\infty}+\|\mu-\bar{\mu}\|\right)\|v\|_{1},
$$

where $C$ denotes (possibly different) positive constants. Therefore, we obtain that

$$
\begin{aligned}
|\langle\mathrm{d} \eta-\mathrm{d} \bar{\eta}, \Phi\rangle| & \leq C\left(\|u-\bar{u}\|_{\infty}+\|\mu-\bar{\mu}\|\right)\|v\|_{1} \\
& \leq C C_{1}\left(\|u-\bar{u}\|_{\infty}+\|\mu-\bar{\mu}\|\right)\|\Phi\|_{2,1} .
\end{aligned}
$$

Consequently, $\|\mathrm{d} \eta-\mathrm{d} \bar{\eta}\|_{2,1 *} \leq C C_{1}\left(\|u-\bar{u}\|_{\infty}+\|\mu-\bar{\mu}\|\right)$, and since by Lemma $4.5 . \| \eta^{2}-$ $\bar{\eta}^{2}\left\|_{\infty} \leq C\right\| \mathrm{d} \eta-\mathrm{d} \bar{\eta} \|_{2,1 *}$, this proves (ii).

Now consider a sequence $\mu_{n} \rightarrow \bar{\mu}$, and $\left(u_{n}, y_{n}\right)$ a stationary point of $\left(\mathcal{P}^{\mu_{n}}\right)$ such that $u_{n} \rightarrow \bar{u}$ in $L^{\infty}$, with (unique) multipliers $\left(p_{n}, \eta_{n}\right)$ and alternative multipliers $\left(p_{n}^{2}, \eta_{n}^{2}\right)$. Since $W^{2,1}(0, T)$ is dense in $C[0, T]$, we deduce easily from point (ii) that $\mathrm{d} \eta_{n} \stackrel{*}{\rightarrow} \mathrm{d} \bar{\eta}$ in $\mathcal{M}[0, T]$, which shows (iii). By the compactness Theorem in BV [1, Th. 3.23], it follows that $\eta_{n}^{1} \rightarrow \bar{\eta}^{1}$ in $L^{1}$, which shows (iv). Finally, since $\eta^{2}$ is given by (2.13), (iv) implies that $\eta_{n}^{2} \rightarrow \bar{\eta}^{2}$ uniformly. By definition (2.14 of $p^{2}$ and Gronwall's Lemma, we conclude that $p_{n}^{2} \rightarrow \bar{p}^{2}$ in $W^{1, \infty}$, which achieves the proof of (v).

\subsection{The uniform second-order growth condition (proof of Prop. 4.2)}

The proof of Prop. 4.2 uses the auxiliary result below. Given $A, B \subset[0, T]$, denote by $\operatorname{exc}\{A, B\}$ the Hausdorff excess of $A$ over $B$, defined by

$$
\operatorname{exc}\{A, B\}:=\sup _{t \in A} \inf _{s \in B}|t-s|,
$$

with the convention $\operatorname{exc}\{\emptyset, B\}=0$. 
Lemma 4.6. Let $\mathrm{d} \bar{\eta} \in \mathcal{M}[0, T]$, and a sequence $\left(\mathrm{d} \eta_{n}\right) \subset \mathcal{M}[0, T]$ be such that $\mathrm{d} \eta_{n}$ weakly-* converges to $\mathrm{d} \bar{\eta}$ in $\mathcal{M}[0, T]$. Then $e_{n}:=\operatorname{exc}\left\{\operatorname{supp}(\mathrm{d} \bar{\eta}), \operatorname{supp}\left(\mathrm{d} \eta_{n}\right)\right\}$ converges to zero when $n \rightarrow+\infty$.

Proof. The result follows from classical compactness arguments. By contradiction, assume that the result is false. Then there exist $\varepsilon_{0}>0$ and a subsequence, still denoted by $\mathrm{d} \eta_{n}$, such that for all $n \in \mathbb{N}^{*}, e_{n}>\varepsilon_{0}$, i.e. there exists $t_{n} \in \operatorname{supp}(\mathrm{d} \bar{\eta})$ such that for all $s \in \operatorname{supp}\left(\mathrm{d} \eta_{n}\right)$, $\left|t_{n}-s\right|>\varepsilon_{0}$. The sequence $\left(t_{n}\right)_{n \in \mathbb{N}^{*}} \subset[0, T]$ being bounded, assume w.l.o.g. that $t_{n} \rightarrow \bar{t} \in$ $[0, T]$. Since $\operatorname{supp}(\mathrm{d} \bar{\eta})$ is closed, $\bar{t} \in \operatorname{supp}(\mathrm{d} \bar{\eta})$. For $n$ large enough, $\left|t_{n}-\bar{t}\right|<\varepsilon_{0} / 2$, and hence, for all $s \in \operatorname{supp}\left(\mathrm{d} \eta_{n}\right),|\bar{t}-s| \geq\left|t_{n}-s\right|-\left|t_{n}-\bar{t}\right|>\varepsilon_{0} / 2$. Let $\varphi$ be a continuous function, with support in $\left[\bar{t}-\varepsilon_{0} / 2, \bar{t}+\varepsilon_{0} / 2\right]$, and such that $\int_{0}^{T} \varphi \mathrm{d} \bar{\eta} \neq 0$. Since $\operatorname{dist}\left\{\bar{t}, \operatorname{supp}\left(\mathrm{d} \eta_{n}\right)\right\}>\varepsilon_{0} / 2$ for all large enough $n, \int_{0}^{T} \varphi \mathrm{d} \eta_{n}=0$. But $\mathrm{d} \eta_{n} \stackrel{*}{\rightarrow} \mathrm{d} \bar{\eta}$, implying that $\int_{0}^{T} \varphi \mathrm{d} \eta_{n} \rightarrow \int_{0}^{T} \varphi \mathrm{d} \bar{\eta}$, which gives the desired contradiction.

Remark 4.7. We may equivalently reformulate Lemma 4.6 as follows: if $\mathrm{d} \eta_{n}$ weakly-* converges to $\mathrm{d} \bar{\eta}$ in $\mathcal{M}[0, T]$, then

$$
\operatorname{supp}(\mathrm{d} \bar{\eta}) \subset \limsup _{n \rightarrow+\infty} \operatorname{supp}\left(\mathrm{d} \eta_{n}\right),
$$

where the limsup is in the sense of Painlevé-Kuratowski.

Proof of Prop. 4.2. We argue by contradiction. If the uniform second-order growth condition does not hold, there exist a stable extension $\left(\mathcal{P}^{\mu}\right)$, a sequence $\mu_{n} \rightarrow \bar{\mu}$, a stationary point $\left(u_{n}, y_{n}\right)$ of $\left(\mathcal{P}^{\mu_{n}}\right)$ such that $u_{n} \rightarrow \bar{u}$ in $L^{\infty}$, with multipliers $\left(p_{n}, \eta_{n}\right)$ and alternative multipliers $\left(p_{n}^{2}, \eta_{n}^{2}\right)$, and a feasible point $\left(\hat{u}_{n}, \hat{y}_{n}\right)$ of $\left(\mathcal{P}^{\mu_{n}}\right)$ such that

$$
J^{\mu_{n}}\left(\hat{u}_{n}\right)<J^{\mu_{n}}\left(u_{n}\right)+o\left(\left\|\hat{u}_{n}-u_{n}\right\|_{2}^{2}\right) .
$$

Introducing the Lagrangian of $\left(\mathcal{P}^{\mu}\right), L^{\mu}(u, \eta)=J^{\mu}(u)+\left\langle\mathrm{d} \eta, G^{\mu}(u)\right\rangle$, and using that $\mathrm{d} \eta_{n} \in$ $N_{K}\left(G^{\mu_{n}}\left(u_{n}\right)\right)$, 4.7) implies that

$$
L^{\mu_{n}}\left(\hat{u}_{n}, \eta_{n}\right)-L^{\mu_{n}}\left(u_{n}, \eta_{n}\right) \leq J^{\mu_{n}}\left(\hat{u}_{n}\right)-J^{\mu_{n}}\left(u_{n}\right)<o\left(\left\|\hat{u}_{n}-u_{n}\right\|_{2}^{2}\right) .
$$

Set $\varepsilon_{n}:=\left\|\hat{u}_{n}-u_{n}\right\|_{2} \rightarrow 0$ and $v_{n}:=\varepsilon_{n}^{-1}\left(\hat{u}_{n}-u_{n}\right)$. A second-order expansion of the Lagrangian shows that $L^{\mu_{n}}\left(\hat{u}_{n}, \eta_{n}\right)-L^{\mu_{n}}\left(u_{n}, \eta_{n}\right)=\varepsilon_{n}^{2} \mathcal{Q}^{\mu_{n}}\left(v_{n}\right)+o\left(\varepsilon_{n}^{2}\right)$, where the quadratic form $\mathcal{Q}^{\mu_{n}}$ is defined like (3.1) for the stationary point $\left(u_{n}, y_{n}\right)$ of $\left(\mathcal{P}^{\mu_{n}}\right)$. Therefore, dividing the above inequality by $\varepsilon_{n}^{2}$, we obtain that

$$
\mathcal{Q}^{\mu_{n}}\left(v_{n}\right) \leq o(1)
$$

Since $\left\|v_{n}\right\|_{2}=1$ for all $n$, taking a subsequence if necessary, we may assume w.l.o.g. that $v_{n} \rightarrow \bar{v}$ weakly in $L^{2}$ for some $\bar{v} \in \mathcal{V}$ when $n \rightarrow+\infty$. Since by Lemma 3.1. $\mathcal{Q}^{\mu_{n}}$ can also be expressed by (3.7), and $\left(u_{n}, y_{n}, p_{n}^{2}, \eta_{n}^{2}\right) \rightarrow\left(\bar{u}, \bar{y}, \bar{p}^{2}, \bar{\eta}^{2}\right)$ uniformly by Prop. 4.4(v), and since $v_{n}$ is bounded in $L^{2}$, it follows that $\mathcal{Q}^{\mu_{n}}\left(v_{n}\right)-\mathcal{Q}\left(v_{n}\right) \rightarrow 0$. Therefore, writing that 
$\mathcal{Q}^{\mu_{n}}\left(v_{n}\right)=\mathcal{Q}\left(v_{n}\right)+\left(\mathcal{Q}^{\mu_{n}}\left(v_{n}\right)-\mathcal{Q}\left(v_{n}\right)\right)$, and using that $\mathcal{Q}$ is a Legendre form and hence weakly l.s.c., we obtain by 4.8 that

$$
\mathcal{Q}(\bar{v}) \leq 0 .
$$

Moreover, since $v_{n} \rightarrow \bar{v}$ weakly in $L^{2}$, and $\left(u_{n}, y_{n}\right) \rightarrow(\bar{u}, \bar{y})$ uniformly, the linearized state $z_{n}$, solution of

$$
\dot{z}_{n}=f_{y}^{\mu_{n}}\left(u_{n}, y_{n}\right) z_{n}+f_{u}^{\mu_{n}}\left(u_{n}, y_{n}\right) v_{n} \quad \text { a.e. on }[0, T], \quad z_{n}(0)=0
$$

converges weakly to $\bar{z}:=z_{\bar{v}}$ in $H^{1}$, and hence uniformly. Since $G^{\mu_{n}}\left(\hat{u}_{n}\right) \in K$, we have that $0 \geq G^{\mu_{n}}\left(\hat{u}_{n}\right)-G^{\mu_{n}}\left(u_{n}\right)=\varepsilon_{n} D G^{\mu_{n}}\left(u_{n}\right) v_{n}+\varepsilon_{n} r_{n}$ on $\operatorname{supp}\left(\mathrm{d} \eta_{n}\right)$, with $\left\|r_{n}\right\|_{\infty}=\mathcal{O}\left(\varepsilon_{n}\right)$. Since $D G^{\mu_{n}}\left(u_{n}\right) v_{n}=g_{y}^{\mu_{n}}\left(y_{n}\right) z_{n}$, it follows that

$$
g_{y}^{\mu_{n}}\left(y_{n}\right) z_{n}+r_{n} \leq 0 \quad \text { on } \operatorname{supp}\left(\mathrm{d} \eta_{n}\right) .
$$

Since $\frac{\mathrm{d}}{\mathrm{d} t} g_{y}^{\mu_{n}}\left(y_{n}(t)\right) z_{n}(t)=\left(g^{\mu_{n}}\right)_{y}^{(1)}\left(y_{n}\right) z_{n}$ is uniformly bounded over $[0, T]$, the functions $g_{y}^{\mu_{n}}\left(y_{n}\right) z_{n}$ are uniformly Lipschitz continuous over $[0, T]$. Therefore,

$$
\begin{aligned}
\sup _{\operatorname{supp}(\mathrm{d} \bar{\eta})} g_{y}(\bar{y}) \bar{z} & \leq\left\|g_{y}(\bar{y}) \bar{z}-g_{y}^{\mu_{n}}\left(y_{n}\right) z_{n}\right\|_{\infty}+\left\|\left(g^{\mu_{n}}\right)_{y}^{(1)}\left(y_{n}\right) z_{n}\right\|_{\infty} e_{n}+\sup _{\operatorname{supp}\left(\mathrm{d} \eta_{n}\right)} g_{y}^{\mu_{n}}\left(y_{n}\right) z_{n} \\
& \leq o(1)+\mathcal{O}\left(e_{n}\right)+\mathcal{O}\left(\varepsilon_{n}\right),
\end{aligned}
$$

where $e_{n}:=\operatorname{exc}\left\{\operatorname{supp}(\mathrm{d} \bar{\eta}), \operatorname{supp}\left(\mathrm{d} \eta_{n}\right)\right\}$ is defined by 4.6). Since $\mathrm{d} \eta_{n} \stackrel{*}{\rightarrow} \mathrm{d} \bar{\eta}$ by Prop. 4.4(iii), it follows from Lemma 4.6 that $e_{n} \rightarrow 0$. Therefore, we obtain that

$$
g_{y}(\bar{y}) \bar{z} \leq 0 \quad \text { on } \operatorname{supp}(\mathrm{d} \bar{\eta}) .
$$

In addition, by (4.7), $D J^{\mu_{n}}\left(u_{n}\right) v_{n} \leq \mathcal{O}\left(\varepsilon_{n}\right)$. Since $D J^{\mu_{n}}\left(u_{n}\right)+D G^{\mu_{n}}\left(u_{n}\right)^{\top} \mathrm{d} \eta_{n}=0$, it follows that $\left\langle\mathrm{d} \eta_{n}, D G^{\mu_{n}}\left(u_{n}\right) v_{n}\right\rangle=\int_{0}^{T} g_{y}^{\mu_{n}}\left(y_{n}\right) z_{n} \mathrm{~d} \eta_{n} \geq \mathcal{O}\left(\varepsilon_{n}\right)$. Since $\mathrm{d} \eta_{n} \stackrel{*}{*} \mathrm{~d} \bar{\eta}$ and $g_{y}^{\mu_{n}}\left(y_{n}\right) z_{n} \rightarrow g_{y}(\bar{y}) \bar{z}$ uniformly, we obtain that $\int_{0}^{T} g_{y}(\bar{y}) \bar{z} \mathrm{~d} \bar{\eta} \geq 0$. Using that $\mathrm{d} \bar{\eta} \geq 0$, 4.11) implies that

$$
g_{y}(\bar{y}) \bar{z}=0 \quad \text { on } \operatorname{supp}(\mathrm{d} \bar{\eta})
$$

i.e. $\bar{v} \in \hat{\mathcal{C}}(\bar{u})$. The strong second-order sufficient condition (3.6) and $(4.9)$ imply then that $\bar{v}=0$. But then $\mathcal{Q}(\bar{v})=0$, and $\mathcal{Q}\left(v_{n}\right) \rightarrow \mathcal{Q}(\bar{v})$ strongly. Since $\mathcal{Q}$ is a Legendre form, we deduce that $v_{n} \rightarrow \bar{v}=0$ strongly in $L^{2}$, contradicting that $\left\|v_{n}\right\|_{2}=1$ for all $n$.

\subsection{The strong regularity framework}

We use the following generalized implicit function theorem in metric spaces by Dontchev and Hager [9], which is a variant of Robinson's strong regularity [27]. 
Theorem 4.8 (9), Th. 2.2). Let $X$ be a complete metric space, $\tilde{X}$ a closed subset of $X, W$ a linear metric space, $\Delta$ a subset of $W, P$ a metric space, $\mathcal{F}: X \times P \rightarrow W, \mathcal{N}: X \rightarrow 2^{W}$, $\mathcal{L}: X \rightarrow W$. Assume that $\mathcal{L}$ is continuous and that there exists $(\bar{x}, \bar{\mu}) \in \tilde{X} \times P$ such that:

(i) $\mathcal{F}(\bar{x}, \bar{\mu}) \in \mathcal{N}(\bar{x})$;

(ii) $\mathcal{F}(\bar{x}, \cdot)$ is continuous at $\bar{\mu}$;

(iii) $\Psi^{\mu}:=\mathcal{F}(\cdot, \mu)-\mathcal{L}(\cdot)$ is strictly stationary at $x=\bar{x}$, uniformly in $\mu$ near $\bar{\mu}$, i.e. for all $\varepsilon>0$, there exists $\nu>0$ such that if $\left\|x_{i}-\bar{x}\right\|_{X},\|\mu-\bar{\mu}\| \leq \nu, i=1,2$,

$$
\left\|\Psi^{\mu}\left(x_{1}\right)-\Psi^{\mu}\left(x_{2}\right)\right\|_{W} \leq \varepsilon\left\|x_{1}-x_{2}\right\|_{X} .
$$

(iv) For all $\delta \in \Delta$, there exists a unique solution $x \in \tilde{X}$ of

$$
\delta \in \mathcal{L}(x)-\mathcal{N}(x),
$$

and there exists $\lambda>0$ such that, with $x_{\delta}$ the unique solution associated with $\delta$,

$$
\left\|x_{\delta}-x_{\delta^{\prime}}\right\|_{X} \leq \lambda\left\|\delta-\delta^{\prime}\right\|_{W}, \quad \forall \delta, \delta^{\prime} \in \Delta .
$$

(v) $\mathcal{F}-\mathcal{L}$ maps a neighborhood of $(\bar{x}, \bar{\mu})$ into $\Delta$.

Then for all $\lambda_{+}>\lambda$, there exist neighborhoods $\mathcal{X}$ of $\bar{x}$ in $\tilde{X}$ and $\mathcal{W}$ of $\bar{\mu}$, such that for each $\mu \in \mathcal{W}$, there exists a unique $x \in \mathcal{X}$ satisfying $\mathcal{F}(x, \mu) \in \mathcal{N}(x)$; moreover, for each $\mu_{i} \in \mathcal{W}$, $i=1,2$, if $x_{i}$ denotes the $x \in \mathcal{X}$ associated with $\mu_{i}$, then

$$
\left\|x_{2}-x_{1}\right\|_{X} \leq \lambda_{+}\left\|\mathcal{F}\left(x_{1}, \mu_{1}\right)-\mathcal{F}\left(x_{1}, \mu_{2}\right)\right\|_{W} .
$$

In [9], the theorem is stated with $\tilde{X}=X$, but remains true if we replace the complete metric space $X$ by any closed subset $\tilde{X}$ of $X$, equipped with the metric of $X$, since $\tilde{X}$ remains a complete metric space.

This theorem was used for stability analysis of optimal control problems subject to firstorder state constraints in 9 . In what follows, we describe a suitable framework to apply Th. 4.8 for second-order state constraints.

Remark 4.9. Our choice of functional spaces to apply Th. 4.8 differs from that of [9] or [18] in the spaces for the state constraint and state constraint multiplier. Whereas in [9, 18, the state constraint is seen in $W^{1, \infty}$, we consider here rather the state constraint in the space of continuous functions $C[0, T]$. Another natural choice for the space of second-order state constraints would be $W^{2, \infty}$ since the constraint is "onto" in this space (Lemma 2.3). The reason for considering here the constraint in $C[0, T]$ is to have multipliers in $\mathcal{M}[0, T]$ instead of in the dual space of $W^{1, \infty}$ or $W^{2, \infty}$. For first-order state constraints it can be shown (see [14]) that the state constraint multiplier $\eta$ lies in $W^{1, \infty}$ (and therefore a suitable

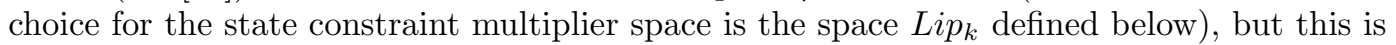
no more true for higher-order state constraints. Note that since $W^{2, \infty} \subset W^{1, \infty} \subset C[0, T]$ with continuous and dense embeddings, and the constraint is "onto" in $W^{2, \infty}$ by Lemma 2.3 the multipliers in the three possible formulations are the same. 
Given $k, l>0$, let

$$
\begin{aligned}
\operatorname{Lip}_{k}(0, T) & :=\left\{u \in W^{1, \infty}(0, T):\|\dot{u}\|_{\infty} \leq k\right\}, \\
B V_{T, l}^{2}[0, T] & :=\left\{\xi \in B V_{T}^{2}[0, T]:|\mathrm{d} \dot{\xi}|_{\mathcal{M}} \leq l\right\},
\end{aligned}
$$

and set

$$
X:=\operatorname{Lip}_{k}\left(0, T ; \mathbb{R}^{m}\right) \times B V_{T, l}^{2}[0, T] .
$$

By Lemma 2.5 we have that $\left(\bar{u}, \bar{\eta}^{2}\right) \in X$ for sufficiently large $k, l$.

Lemma 4.10. Equipped with the norm

$$
\|(u, \xi)\|_{X}:=\|u\|_{2}+\|\xi\|_{2},
$$

$X$ is a complete metric space, and

$$
\|u\|_{\infty} \leq \max \left\{\sqrt{3 / T}\|u\|_{2}, \sqrt[3]{3 k}\|u\|_{2}^{2 / 3}\right\}, \quad \text { for all } u \in \operatorname{Lip}_{k}(0, T) .
$$

Proof. It was shown in [9, Lemma 3.2] that the space $\left(\operatorname{Lip}_{k}(0, T),\|\cdot\|_{2}\right)$ is a complete metric space, and the estimate (4.17) follows from [9, Lemma 3.1]. We show now that $\left(B V_{T, l}^{2}[0, T],\|\cdot\|_{2}\right)$ is complete as well. Let $\left(\xi_{n}\right)$ be a Cauchy sequence in $B V_{T, l}^{2}[0, T]$ (for the norm $\left.\|\cdot\|_{2}\right)$. Since $L^{2}(0, T)$ is complete, there exists $\tilde{\xi} \in L^{2}(0, T)$ such that $\xi_{n} \rightarrow \tilde{\xi}$ in $L^{2}$. Let us show that the limit point $\tilde{\xi}$ lies in $B V_{T, l}^{2}[0, T]$. We have that $\left|\mathrm{d} \dot{\xi}_{n}\right|_{\mathcal{M}} \leq l$ for all $n$, and since $\dot{\xi}_{n}(T)=0$, the sequence $\left(\dot{\xi}_{n}\right)$ is bounded in BV for the norm $\|\eta\|_{B V}:=\|\eta\|_{1}+|\mathrm{d} \eta|_{\mathcal{M}}$. Therefore, by the compactness theorem in BV [1, Th. 3.23], there exists a subsequence $\xi_{\psi(n)}$ and $\zeta \in B V[0, T]$ such that $\mathrm{d} \dot{\xi}_{\psi(n)}{ }^{*} \mathrm{~d} \zeta$ weakly-* in $\mathcal{M}[0, T]$ and $\dot{\xi}_{\psi(n)} \rightarrow \zeta$ in $L^{1}$. Moreover, using the integration by parts formula in BV [12, p.154], we obtain that

$$
T \zeta(T)=\int_{0}^{T}\left(\zeta(t)-\dot{\xi}_{\psi(n)}(t)\right) \mathrm{d} t+\int_{0}^{T} s\left(\mathrm{~d} \zeta(s)-\mathrm{d} \dot{\xi}_{\psi(n)}(s)\right) \rightarrow 0,
$$

and hence $\zeta(T)=0$. Setting $\hat{\xi}(t):=-\int_{t}^{T} \zeta(s) \mathrm{d} s$, we have that $\hat{\xi} \in B V_{T}^{2}[0, T]$, and $\xi_{\psi(n)} \rightarrow \hat{\xi}$ in $L^{\infty}$ and a fortiori in $L^{2}$. We deduce that necessarily, $\hat{\xi}=\tilde{\xi} \in B V_{T}^{2}[0, T]$, the whole sequence $\left(\mathrm{d} \dot{\xi}_{n}\right)$ weakly-* converges to d $\dot{\tilde{\xi}}$ in $\mathcal{M}[0, T]$, and then

$$
|\mathrm{d} \dot{\tilde{\xi}}|_{\mathcal{M}} \leq \liminf \left|\mathrm{d} \dot{\xi}_{n}\right|_{\mathcal{M}} \leq l
$$

This shows that $\tilde{\xi} \in B V_{T, l}^{2}[0, T]$, and hence, $\left(B V_{T, l}^{2}[0, T],\|\cdot\|_{2}\right)$ is a complete metric space. This achieves the proof.

Note that for all $\xi \in B V_{T, l}^{2}[0, T]$, we have that $|\mathrm{d} \dot{\xi}|_{\mathcal{M}} \leq l$, and since $\dot{\xi}(T)=0$, it follows that $\|\dot{\xi}\|_{\infty} \leq l$, and hence, $B V_{T, l}^{2}[0, T] \subset \operatorname{Lip}_{l}(0, T)$. Therefore, we deduce from 4.17) that

$$
\|\xi\|_{\infty} \leq \max \left\{\sqrt{3 / T}\|\xi\|_{2}, \sqrt[3]{3 l}\|\xi\|_{2}^{2 / 3}\right\}, \quad \text { for all } \xi \in B V_{T, l}^{2}[0, T] .
$$


Given $r>0$, let

$$
\tilde{X}:=\left\{x=(u, \xi) \in X:\|u-\bar{u}\|_{2} \leq r\right\} .
$$

Since $\tilde{X}$ is a closed subset of $X$, by Lemma 4.10 the space $\tilde{X}$ equipped with the norm of $X$ (4.16) is a complete metric space. We need to work with $\tilde{X}$ instead of $X$ in order to obtain the uniqueness of a solution of 4.13 in $\tilde{X}$, for small enough $r>0$.

Let $P$ denotes a closed neighborhood of $\bar{\mu}$, contained in $M_{0}$, and let

$$
W:=L^{2}\left(0, T ; \mathbb{R}^{m *}\right) \times H^{2}(0, T),
$$

equipped with the norm $\|\delta\|_{W}:=\|\gamma\|_{2}+\|\zeta\|_{2,2}$ for $\delta=(\gamma, \zeta) \in W$. Given a stable extension $\left(\mathcal{P}^{\mu}\right)$ of $(\mathcal{P})$, our formulation is the following: For $\mu$ in the neighborhood of $\bar{\mu}$, find $x=$ $\left(u, \eta^{2}\right) \in \tilde{X}$ solution of

$$
\mathcal{F}(x, \mu) \in \mathcal{N}(x),
$$

where $\mathcal{F}$ and $\mathcal{N}$ are as follows:

- $\mathcal{F}: X \times P \rightarrow W$,

$$
\mathcal{F}(x, \mu):=\left(\begin{array}{c}
\tilde{H}_{u}^{\mu}\left(u, y_{u}^{\mu}, p_{u, \mu}^{2, \mu}, \eta^{2}\right) \\
g^{\mu}\left(y_{u}^{\mu}\right)
\end{array}\right),
$$

where $\tilde{H}^{\mu}$ is the alternative Hamiltonian $(2.16)$ of $\left(\mathcal{P}^{\mu}\right), y_{u}^{\mu}$ is the solution of the state equation 2.28) and $p_{u, \eta^{2}}^{2, \mu}$ is the solution of the alternative costate equation 2.17) for $\left(\mathcal{P}^{\mu}\right)$, i.e.:

$$
-\dot{p}_{u, \eta^{2}}^{2, \mu}=\tilde{H}_{y}^{\mu}\left(u, y_{u}^{\mu}, p_{u, \eta^{2}}^{2, \mu}, \eta^{2}\right) \quad \text { a.e. on }[0, T], \quad p_{u, \eta^{2}}^{2, \mu}(T)=\phi_{y}^{\mu}\left(y_{u}^{\mu}(T)\right) .
$$

- $\mathcal{N}: X \rightarrow 2^{W}, \mathcal{N}(x)=\{0\} \times\left(N_{K^{-}}\left(\mathrm{d} \dot{\eta}^{2}\right) \cap H^{2}(0, T)\right)$, where

$$
N_{K^{-}}\left(\mathrm{d} \dot{\eta}^{2}\right)= \begin{cases}\left\{\varphi \in C_{-}[0, T]:\left\langle\mathrm{d} \dot{\eta}^{2}, \varphi\right\rangle=0\right\} & \text { if } \mathrm{d} \dot{\eta}^{2} \geq 0 \\ \emptyset & \text { otherwise. }\end{cases}
$$

Then $\left(u, y_{u}^{\mu}\right)$ is a stationary point of $\left(\mathcal{P}^{\mu}\right)$ with alternative multipliers $\left(p_{u, \eta^{2}}^{2, \mu}, \eta^{2}\right)$ iff $x=$ $\left(u, \eta^{2}\right)$ is solution of 4.21.

The space of sufficiently smooth variations $\Delta \subset W$, in assumptions (iv) and (v) of Th. 4.8 is, for some $\varrho, k^{\prime}>0$,

$$
\Delta:=\left\{\delta \in \operatorname{Lip}_{k^{\prime}}\left(0, T ; \mathbb{R}^{m *}\right) \times H^{2}(0, T),\|\delta\|_{W} \leq \varrho\right\} .
$$

Finally, Theorem 4.8 is applied with

$$
\mathcal{L}(x):=\mathcal{F}(\bar{x}, \bar{\mu})-D_{x} \mathcal{F}(\bar{x}, \bar{\mu})(x-\bar{x}) .
$$

We have now defined all the elements to apply Th. 4.8 to prove Th. 4.3 in section 6 . 


\section{Stability analysis of linear-quadratic problems}

The verification of assumption (iv) of Th. 4.8 is strongly related to stability analysis of linearquadratic optimal control problems with a second-order state constraint, that we study in this section. Since these results have their own interest, they are stated independently of the rest of the paper. The problem under consideration is of the form:

$$
\begin{aligned}
\left(\mathcal{P}_{\delta}\right) \min _{(v, z) \in \mathcal{V} \times \mathcal{Z}} & \frac{1}{2} \int_{0}^{T}\left(v(t)^{\top} S(t) v(t)+2 v(t)^{\top} R(t) z(t)+z(t)^{\top} Q(t) z(t)\right) \mathrm{d} t \\
& +\int_{0}^{T}(a(t) z(t)+(b(t)-\gamma(t)) v(t)) \mathrm{d} t+\frac{1}{2} z(T)^{\top} \Phi z(T) \\
& \\
\text { s.t. } \quad & \dot{z}(t)=A(t) z(t)+B(t) v(t) \quad \text { a.e. on }[0, T], \quad z(0)=0 \\
& C(t) z(t)+d(t)-\zeta(t) \leq 0 \quad \text { on }[0, T] .
\end{aligned}
$$

The perturbation parameter is here $\delta=(\gamma, \zeta) \in W=L^{2}\left(0, T ; \mathbb{R}^{m *}\right) \times H^{2}(0, T)$, with the norm $\|\delta\|_{W}=\|\gamma\|_{2}+\|\zeta\|_{2,2}$. The control and state spaces for the linearized problem are $\mathcal{V}:=L^{2}\left(0, T ; \mathbb{R}^{m}\right)$ and $\mathcal{Z}:=H^{1}\left(0, T ; \mathbb{R}^{n}\right)$. The state constraint (5.4) is scalar-valued. The matrix and vectors $S(\cdot), R(\cdot), Q(\cdot), a(\cdot), b(\cdot), A(\cdot), B(\cdot), C(\cdot), d(\cdot)$, of appropriate dimensions, are Lipschitz continuous functions of time. In addition, $C(\cdot)$ and $d(\cdot)$ lie in the space $W^{3, \infty}$ and $A(\cdot)$ in $W^{2, \infty}$. The matrix $S$ and $Q$ are symmetric. We assume in addition in all this section that (recall (A1))

$$
d(0)<0 .
$$

Given $v \in \mathcal{V}$, we denote by $z_{v}$ the unique solution in $\mathcal{Z}$ of the linearized state equation $(5.3)$. Then we may write $\left(\mathcal{P}_{\delta}\right)$ as follows:

$$
\left(\mathcal{P}_{\delta}\right) \quad \min _{v \in \mathcal{V}} \mathcal{J}^{\delta}(v), \quad \Gamma^{\delta}(v) \in K
$$

with $\mathcal{J}^{\delta}(v):=\int_{0}^{T}\left\{\frac{1}{2}\left(v^{\top} S v+2 v^{\top} R z_{v}+z_{v}^{\top} Q z_{v}\right)+a z+(b-\gamma) v\right\} \mathrm{d} t+\frac{1}{2} z_{v}(T)^{\top} \Phi z_{v}(T), \Gamma^{\delta}(v):=$ $C z_{v}+d-\zeta$ and $K=C_{-}[0, T]$.

Assume that $C(t) B(t) \equiv 0$ on $[0, T]$ (state constraint of second-order), and define the matrix:

$$
C_{1}(t):=\dot{C}(t)+C(t) A(t), \quad C_{2}(t):=\dot{C}_{1}(t)+C_{1}(t) A(t), \quad N_{2}(t):=C_{1}(t) B(t) .
$$

Then for all $v \in \mathcal{V}$, we have that

$$
\frac{\mathrm{d}}{\mathrm{d} t}\left\{C(t) z_{v}(t)\right\}=C_{1}(t) z_{v}(t), \quad \frac{\mathrm{d}^{2}}{\mathrm{~d} t^{2}}\left\{C(t) z_{v}(t)\right\}=C_{2}(t) z_{v}(t)+N_{2}(t) v(t) .
$$

The alternative multipliers $\left(\pi^{2}, \eta^{2}\right) \in W^{1, \infty}\left(0, T ; \mathbb{R}^{n *}\right) \times B V_{T}^{2}[0, T]$ for the linear-quadratic problem are defined by

$$
\begin{aligned}
\eta^{1}(t) & :=-\int_{] t, T]} \mathrm{d} \eta(s), \quad \eta^{2}(t):=\int_{t}^{T} \eta^{1}(s) \mathrm{d} s \\
\pi^{2}(t) & :=\pi(t)-\eta^{1}(t) C(t)-\eta^{2}(t) C_{1}(t), \quad t \in[0, T] .
\end{aligned}
$$


Let $\left(\bar{v}, \bar{z}=z_{\bar{v}}\right)$ be a stationary point of $\left(\mathcal{P}_{0}\right)$, with multipliers $(\bar{\pi}, \bar{\eta})$ and alternative multipliers $\left(\bar{\pi}^{2}, \bar{\eta}^{2}\right)$. Denote the contact set by $\Omega:=\{t \in[0, T]: C(t) \bar{z}(t)+d(t)=0\}$, and a neighborhood of the contact set by $\Omega_{\sigma}:=\{t \in[0, T]$ : $\operatorname{dist}\{t, \Omega\}<\sigma\}$ for $\sigma>0$. For linear-quadratic problems, assumptions (A2)-(A3) may be rewritten as follows:

$(\tilde{A} 2)$ The state constraint is a regular second-order state constraint, i.e. $C(t) B(t) \equiv 0$ on $[0, T]$, and there exists $\beta, \sigma>0$ such that

$$
\left|N_{2}(t)\right| \geq \beta \quad \text { on } \Omega_{\sigma} .
$$

(A3) The matrix $S(t)$ is uniformly positive definite over $[0, T]$, i.e.,

$$
\exists \alpha>0, \quad v^{\top} S(t) v \geq \alpha|v|^{2}, \quad \text { for all } t \in[0, T] \text { and all } v \in \mathbb{R}^{m} .
$$

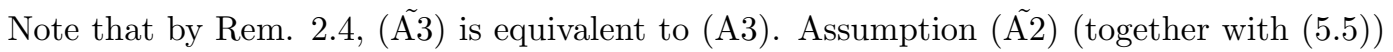
imply the following (cf Lemma 2.3):

Lemma 5.1. Assume that (Ã2) holds. Then for all $\varepsilon \in(0, \sigma)$, with the $\sigma$ of $(\tilde{\mathrm{A} 2})$, so small that 2.23 holds, there exists a positive constant $c$ such that for all $\varphi \in H^{2}(0, T)$, there exists $v \in \mathcal{V}$ satisfying

$$
C(t) z_{v}(t)=\varphi(t) \quad \text { on } \Omega_{\varepsilon} \quad \text { and } \quad\|v\|_{2} \leq c\|\varphi\|_{2,2} .
$$

Therefore (Ã2) (and (5.5) imply that Robinson's constraint qualification holds, and that the multipliers associated with $(\bar{v}, \bar{z})$ are unique.

Propositions 5.2 and 5.3 below hold for a larger set of perturbations, more precisely for $\delta=(\gamma, \zeta) \in \hat{W}$, where

$$
\hat{W}:=L^{2}\left(0, T ; \mathbb{R}^{m}\right) \times C[0, T],
$$

equipped with its standard norm $\|\delta\|_{\hat{W}}:=\|\gamma\|_{2}+\|\zeta\|_{\infty}$. We have of course $W \subset \hat{W}$ with continuous embedding. Identically to Prop. 4.4, we obtain the stability of multipliers for linear-quadratic problems (with a slightly modified statement).

Proposition 5.2. Let $(\bar{v}, \bar{z})$ be a stationary point of $\left(\mathcal{P}_{0}\right)$ satisfying $(\tilde{\mathrm{A}} 2)$. Then there exists $\nu>0$ such that for every stationary point $(v, z)$ of $\left(\mathcal{P}_{\delta}\right)$, with (unique) multipliers $(\pi, \eta)$ and alternative multipliers $\left(\pi^{2}, \eta^{2}\right)$ defined by (5.7)-(5.6), the following hold:

(i) If $\|\delta\|_{\hat{W}},\|v-\bar{v}\|_{2}<\nu$, then $\mathrm{d} \eta$ is uniformly bounded in $\mathcal{M}[0, T]$;

(ii) There exists $\kappa>0$ such that, for all $\|\delta\|_{\hat{W}},\|v-\bar{v}\|_{2}<\nu$,

$$
\|\mathrm{d} \eta-\mathrm{d} \bar{\eta}\|_{2,2 *},\left\|\eta^{2}-\bar{\eta}^{2}\right\|_{2} \leq \kappa\left(\|v-\bar{v}\|_{2}+\|\delta\|_{\hat{W}}\right)
$$

Moreover, when $\|\delta\|_{\hat{W}},\|v-\bar{v}\|_{2} \rightarrow 0$ :

(iii) $\mathrm{d} \eta$ weakly- * converges to $\mathrm{d} \bar{\eta}$ in $\mathcal{M}[0, T]$;

(iv) $\eta^{1} \rightarrow \bar{\eta}^{1}$ in $L^{1}$;

(v) $\pi^{2}$ and $\eta^{2}$ converges uniformly to $\bar{\pi}^{2}$ and $\bar{\eta}^{2}$, respectively.

RR $n^{\circ} 0123456789$ 


\section{Second-order optimality conditions}

Let $\tilde{\mathcal{Q}}$ denote the quadratic part of the cost $\mathcal{J}^{\delta}$ (independent of $\delta$ ):

$$
\begin{aligned}
\tilde{\mathcal{Q}}(v)= & \frac{1}{2} \int_{0}^{T}\left(v(t)^{\top} S(t) v(t)+2 v(t)^{\top} R(t) z_{v}(t)+z_{v}(t)^{\top} Q(t) z_{v}(t)\right) \mathrm{d} t \\
& +\frac{1}{2} z_{v}(T)^{\top} \Phi z_{v}(T) .
\end{aligned}
$$

The strong second-order sufficient condition is:

$$
\tilde{\mathcal{Q}}(v)>0, \quad \text { for all } v \in \mathcal{V} \backslash\{0\} \text { such that } C(t) z_{v}(t)=0 \text { on } \operatorname{supp}(\mathrm{d} \bar{\eta}) \text {. }
$$

Identically to Prop. 4.2, we obtain that the second-order sufficient condition 5.10 implies the uniform second-order growth condition for the perturbed problems $\left(\mathcal{P}_{\delta}\right)$ (here again the statement is slightly modified).

Proposition 5.3. Let $(\bar{v}, \bar{z})$ be a stationary point of $\left(\mathcal{P}_{0}\right)$ satisfying $(\tilde{\mathrm{A}} 2)-(\tilde{\mathrm{A}} 3)$ and the strong second-order sufficient condition (5.10). Then there exist $c, \rho>0$ and a neighborhood $\mathcal{W}$ of 0 in $\hat{W}$, such that for all $\delta \in \mathcal{W}$ and any stationary point $\left(v_{\delta}, z_{\delta}\right)$ of $\left(\mathcal{P}_{\delta}\right)$ with $\left\|v_{\delta}-\bar{v}\right\|_{2}<\rho$,

$$
\mathcal{J}^{\delta}(v) \geq \mathcal{J}^{\delta}\left(v_{\delta}\right)+c\left\|v-v_{\delta}\right\|_{2}^{2}, \quad \forall v \in \mathcal{V}: \Gamma^{\delta}(v) \in K,\|v-\bar{v}\|_{2}<\rho .
$$

\section{Stability Analysis}

The main result of this section is the theorem below. The key point to show the existence of a stationary point for the perturbed linear-quadratic problem under the weak second-order sufficient condition 5.10 taking into account the active constraints is the uniform growth condition (Prop. 5.3) and a theorem from abstract optimization theory by Bonnans and Shapiro [6, Th. 5.17 and Rem. 5.19].

Theorem 5.4. Let $(\bar{v}, \bar{z})$ be a stationary point of $\left(\mathcal{P}_{0}\right)$ satisfying $(\tilde{\mathrm{A}} 2)-(\tilde{\mathrm{A}} 3)$ and the strong second-order sufficient condition (5.10). Then there exist $c, \rho, \lambda>0$ and a neighborhood $\mathcal{W}$ of 0 in $W$, such that for all $\delta \in \mathcal{W},\left(\mathcal{P}_{\delta}\right)$ has a unique stationary point $\left(v_{\delta}, z_{v_{\delta}}\right)$ with $\left\|v_{\delta}-\bar{v}\right\|_{2}<\rho$ and unique associated alternative multipliers $\left(\pi_{\delta}^{2}, \eta_{\delta}^{2}\right)$, and

$$
\left\|v_{\delta}-v_{\delta^{\prime}}\right\|_{2}+\left\|\eta_{\delta}^{2}-\eta_{\delta^{\prime}}^{2}\right\|_{2} \leq \lambda\left\|\delta-\delta^{\prime}\right\|_{W}, \quad \forall \delta, \delta^{\prime} \in \mathcal{W} .
$$

Moreover, $\left(v_{\delta}, z_{v_{\delta}}\right)$ is a local solution of $\left(\mathcal{P}_{\delta}\right)$ satisfying the uniform quadratic growth condition (5.11).

Proof. Let us show the existence of a stationary point of problem $\left(\mathcal{P}_{\delta}\right)$. We may write $\left(\mathcal{P}_{\delta}\right)$ as

$$
\left(\mathcal{P}_{\delta}\right) \quad \min _{v \in \mathcal{V}} \frac{1}{2}\langle v, \mathcal{A} v\rangle+\langle b, v\rangle-\langle\gamma, v\rangle \quad \text { s.t. } \quad \mathcal{C} v+d-\zeta \in K
$$

where $\mathcal{A}$ is the continuous, self-adjoint bilinear operator over $\mathcal{V}$ associated with the quadratic form $5.9, b$ is an element in $\mathcal{V}^{*} \equiv \mathcal{V}, \mathcal{C}: v \mapsto C z_{v}$ is a linear continuous operator $\mathcal{V} \rightarrow$ 
$C[0, T]$, and $d \in H^{2}(0, T)$. Here, without ambiguity, we also denote by $\langle\cdot, \cdot\rangle$ the scalar product over $\mathcal{V}$.

Step 1: Reduction to a fixed feasible set. Let us first consider perturbations of the cost function only, i.e. consider the problem $\left(\mathcal{P}_{\gamma}\right)$ defined by

$$
\left(\mathcal{P}_{\gamma}\right) \min _{v \in \mathcal{V}} \frac{1}{2}\langle v, \mathcal{A} v\rangle+\langle b, v\rangle-\langle\gamma, v\rangle \quad \text { s.t. } \quad \mathcal{C} v+d \in K .
$$

By Prop. 5.3 the uniform second-order growth condition holds for $\left(\mathcal{P}_{\gamma}\right)$, so does Robinson's constraint qualification by $(\tilde{\mathrm{A} 2})$, and the perturbed problem $\left(\mathcal{P}_{\gamma}\right)$ includes the so-called tilt perturbation (see [6, p.416]), i.e. additive perturbations of the cost function of type $-\langle\gamma, v\rangle$ with $\gamma \in \mathcal{V}^{*}$. Therefore, it follows from [6. Th. 5.17 and Rem. 5.19], since the feasible set of $\left(\mathcal{P}_{\gamma}\right)$ is constant, that there exist $\rho_{1}, \rho_{2}>0$ and a constant $\lambda>0$, such that for all $\gamma \in B_{2}\left(0, \rho_{2}\right),\left(\mathcal{P}_{\gamma}\right)$ has a unique stationary point $v_{\gamma}$ in $B_{2}\left(\bar{v}, \rho_{1}\right)$, and

$$
\left\|v_{\gamma}-v_{\gamma^{\prime}}\right\|_{2} \leq \lambda\left\|\gamma-\gamma^{\prime}\right\|_{2}, \quad \forall \gamma, \gamma^{\prime} \in B_{2}\left(0, \rho_{2}\right) .
$$

We have of course that $\bar{v}=v_{0}$.

Step 2: Existence of a stationary point of $\left(\mathcal{P}_{\delta}\right)$. Fix $\varepsilon \in(0, \sigma)$ with the $\sigma$ of (A2) satisfying 2.23). Let now $\delta=(\gamma, \zeta) \in W$. By Lemma 5.1. there exists $v_{\zeta} \in \mathcal{V}$ such that

$$
\left(\mathcal{C} v_{\zeta}\right)(t)=\zeta(t) \quad \text { on } \Omega_{\varepsilon} \quad \text { and } \quad\left\|v_{\zeta}\right\|_{2} \leq c\|\zeta\|_{2,2} .
$$

Set $\tilde{\gamma}:=\gamma-\mathcal{A} v_{\zeta}$. We have that $\|\tilde{\gamma}\|_{2} \leq\|\gamma\|_{2}+c\|\mathcal{A}\|\|\zeta\|_{2,2}<\rho_{2}$ if $\|\delta\|_{W}$ is small enough. Therefore, there exists a (unique) stationary point $v_{\tilde{\gamma}} \in B_{2}\left(\bar{v}, \rho_{1}\right)$ of $(\mathcal{P} \tilde{\gamma})$, with multiplier $\mathrm{d} \eta_{\tilde{\gamma}} \in \mathcal{M}[0, T]$, satisfying the first-order optimality condition

$$
\left\{\begin{array}{l}
\mathcal{A} v_{\tilde{\gamma}}+b-\tilde{\gamma}+\mathcal{C}^{\top} \mathrm{d} \eta_{\tilde{\gamma}}=0 \\
\mathcal{C} v_{\tilde{\gamma}}+d \leq 0 \text { on }[0, T], \quad \mathrm{d} \eta_{\tilde{\gamma}} \geq 0, \quad\left\langle\mathrm{~d} \eta_{\tilde{\gamma}}, \mathcal{C} v_{\tilde{\gamma}}+d\right\rangle=0
\end{array}\right.
$$

Since $\left\|\mathcal{C} v_{\tilde{\gamma}}-\mathcal{C} \bar{v}\right\|_{\infty} \leq\|\mathcal{C}\|\left\|v_{\tilde{\gamma}}-\bar{v}\right\|_{2} \leq \lambda\|\mathcal{C}\|\|\tilde{\gamma}\|_{2}$ by (5.13), if $\|\delta\|_{W}$ is small enough then the contact set of $\mathcal{C} v_{\tilde{\gamma}}+d$ is included in $\Omega_{\varepsilon}$, and hence

$$
\operatorname{supp}\left(\mathrm{d} \eta_{\tilde{\gamma}}\right) \subset \Omega_{\varepsilon} .
$$

Let $v_{\delta}:=v_{\tilde{\gamma}}+v_{\zeta}$ and $\mathrm{d} \eta_{\delta}:=\mathrm{d} \eta_{\tilde{\gamma}}$. Note that there exists a constant $a>0$ such that $(\mathcal{C} \bar{v})(t)+d(t)<-a$ on $[0, T] \backslash \Omega_{\varepsilon}$. Therefore, on $[0, T] \backslash \Omega_{\varepsilon}$, we obtain that (we denote in what follows by $C$ different positive constants)

$$
\begin{aligned}
\mathcal{C} v_{\delta}+d-\zeta & =\mathcal{C} \bar{v}+d-\zeta+\mathcal{C} v_{\zeta}+\mathcal{C}\left(v_{\tilde{\gamma}}-\bar{v}\right) \\
& \leq-a+\|\zeta\|_{\infty}+\left\|\mathcal{C} v_{\zeta}\right\|_{\infty}+\left\|\mathcal{C}\left(v_{\tilde{\gamma}}-\bar{v}\right)\right\|_{\infty} \\
\leq & -a+C\|\zeta\|_{2,2}+\|\mathcal{C}\|\left\|v_{\zeta}\right\|_{2}+\|\mathcal{C}\|\left\|v_{\tilde{\gamma}}-\bar{v}\right\|_{2} \\
\leq & -a+(C+c\|\mathcal{C}\|)\|\zeta\|_{2,2}+\lambda\|\mathcal{C}\|\|\tilde{\gamma}\|_{2} \leq-a+C\|\delta\|_{W}
\end{aligned}
$$

RR $n^{\circ} 0123456789$ 
and hence, if $\|\delta\|_{W}$ is small enough, then $\mathcal{C} v_{\delta}+d-\zeta<0$ on $[0, T] \backslash \Omega_{\varepsilon}$. Since on $\Omega_{\varepsilon}$, we have that $\mathcal{C} v_{\delta}+d-\zeta=\mathcal{C} v_{\tilde{\gamma}}+d \leq 0$, using (5.14) and (5.15), $v_{\delta}$ obviously satisfies

$$
\left\{\begin{array}{l}
\mathcal{A} v_{\delta}+b-\gamma+\mathcal{C}^{\top} \mathrm{d} \eta_{\delta}=0, \\
\mathcal{C} v_{\delta}+d-\zeta \leq 0 \text { on }[0, T], \quad \mathrm{d} \eta_{\delta} \geq 0, \quad\left\langle\mathrm{~d} \eta_{\delta}, \mathcal{C} v_{\delta}+d-\zeta\right\rangle=0,
\end{array}\right.
$$

i.e. $v_{\delta}$ is a stationary point of $\left(\mathcal{P}_{\delta}\right)$, with multiplier $\mathrm{d} \eta_{\delta}$. Consequently, for $\rho_{3}>0$ small enough, reducing $\rho_{1}$ if necessary, $\left(\mathcal{P}_{\delta}\right)$ has, for all $\delta \in B_{W}\left(0, \rho_{3}\right)$, a (necessarily unique by Prop. 5.3 stationary point $v_{\delta} \in B_{2}\left(\bar{v}, \rho_{1}\right)$, with (unique) multiplier $\mathrm{d} \eta_{\delta}$. That $\left(v_{\delta}, z_{v_{\delta}}\right)$ is a local solution of $\left(\mathcal{P}_{\delta}\right)$ satisfying the uniform growth condition 5.11 follows then from Prop. 5.3 .

Step 3: Lipschitz continuity of the stationary point. Let $\delta_{i}=\left(\gamma_{i}, \zeta_{i}\right) \in B_{W}\left(0, \rho_{3}\right)$, $i=1,2$, and $v_{\zeta_{i}}$ be such that

$$
\mathcal{C} v_{\zeta_{i}}=\zeta_{i} \text { on } \Omega_{\varepsilon}, i=1,2, \quad \text { and } \quad\left\|v_{\zeta_{1}}\right\|_{2} \leq c\left\|\zeta_{1}\right\|_{2,2},\left\|v_{\zeta_{1}}-v_{\zeta_{2}}\right\|_{2} \leq c\left\|\zeta_{1}-\zeta_{2}\right\|_{2,2} \text {. }
$$

It follows that $\left\|v_{\zeta_{2}}\right\|_{2} \leq c\left(2\left\|\zeta_{1}\right\|_{2,2}+\left\|\zeta_{2}\right\|_{2,2}\right)<3 c \rho_{3}$. Setting $\tilde{\gamma}_{i}:=\gamma_{i}-\mathcal{A} v_{\zeta_{i}}$, we obtain as before that if $\rho_{3}$ is small enough, then the unique stationary point $v_{i}$ of $\left(\mathcal{P}_{\delta_{i}}\right)$ is given by $v_{i}=v_{\zeta_{i}}+v_{\tilde{\gamma}_{i}}$. Therefore, using (5.13,

$$
\begin{aligned}
\left\|v_{1}-v_{2}\right\|_{2} & \leq\left\|v_{\zeta_{1}}-v_{\zeta_{2}}\right\|_{2}+\lambda\left\|\tilde{\gamma}_{1}-\tilde{\gamma}_{2}\right\|_{2} \\
& \leq c(1+\lambda\|\mathcal{A}\|)\left\|\zeta_{1}-\zeta_{2}\right\|_{2,2}+\lambda\left\|\gamma_{1}-\gamma_{2}\right\|_{2} \\
& \leq C\left\|\delta_{1}-\delta_{2}\right\|_{W} .
\end{aligned}
$$

Step 4: Lipschitz continuity of the alternative multiplier $\eta_{\delta}^{2}$ given by 5.6 . Using the above notation, denote by $\mathrm{d} \eta_{i}$ the (unique) multiplier associated with $v_{i}$ and by $\eta_{i}^{2}$ the associated alternative multiplier. Since $-\mathcal{C}^{\top}\left(\mathrm{d} \eta_{2}-\mathrm{d} \eta_{1}\right)=\mathcal{A}\left(v_{2}-v_{1}\right)+\gamma_{2}-\gamma_{1}$, we have, for all $v \in \mathcal{V}$,

$$
\left|\left\langle\mathrm{d} \eta_{2}-\mathrm{d} \eta_{1}, \mathcal{C} v\right\rangle\right| \leq\left(\|\mathcal{A}\|\left\|v_{2}-v_{1}\right\|_{2}+\left\|\gamma_{2}-\gamma_{1}\right\|_{2}\right)\|v\|_{2}
$$

By Lemma 5.1. for all $\varphi \in H^{2}(0, T)$, there exists $v \in \mathcal{V}$ such that $\mathcal{C} v=\varphi$ on $\Omega_{\varepsilon}$ and $\|v\|_{2} \leq c\|\varphi\|_{2,2}$. It follows from (5.15) that $\int_{0}^{T} \varphi(t)\left(\mathrm{d} \eta_{2}(t)-\mathrm{d} \eta_{1}(t)\right)=\left\langle\mathrm{d} \eta_{2}-\mathrm{d} \eta_{1}, \mathcal{C} v\right\rangle$. Therefore, we obtain in view of (5.17) that

$$
\left\|\mathrm{d} \eta_{2}-\mathrm{d} \eta_{1}\right\|_{2,2 *}=\sup _{\varphi \in H^{2}, \varphi \neq 0} \frac{\left|\int_{0}^{T} \varphi(t)\left(\mathrm{d} \eta_{2}(t)-\mathrm{d} \eta_{1}(t)\right)\right|}{\|\varphi\|_{2,2}} \leq c\left(\|\mathcal{A}\|\left\|v_{2}-v_{1}\right\|_{2}+\left\|\gamma_{2}-\gamma_{1}\right\|_{2}\right) .
$$

Since $\left\|\eta_{2}^{2}-\eta_{1}^{2}\right\|_{2} \leq C\left\|\mathrm{~d} \eta_{2}-\mathrm{d} \eta_{1}\right\|_{2,2 *}$ by Lemma 4.5 and with (5.16), this shows the existence of a constant $\lambda>0$ such that $(5.12)$ holds and achieves the proof of the theorem.

\section{Proof of Theorem 4.3}

In order to prove Th. 4.3. we have to show that assumptions (iii), (iv) and (v) of Th. 4.8 are satisfied, which is done respectively in lemmas 6.1 to 6.3 below. In all this section, the 
assumptions of Th. 4.3 are assumed to hold. We consider a stable extension $\left(\mathcal{P}^{\mu}\right)$ of $(\mathcal{P})$, and we use the notations defined in subsection 4.3 .

Let us first make explicit the expression of the derivative $D_{x} \mathcal{F}(\bar{x}, \bar{\mu})(x-\bar{x})$ involved in the definition (4.24) of $\mathcal{L}(x)$, with $x=\left(u, \eta^{2}\right)$ and $\bar{x}=\left(\bar{u}, \bar{\eta}^{2}\right)$. Note that the Fréchet derivative of the mapping $(u, \mu) \mapsto y_{u}^{\mu}$ w.r.t. $u$ in direction $v$ is the solution $z_{u, v}^{\mu}$ of

$$
\dot{z}_{u, v}^{\mu}=f_{y}^{\mu}\left(u, y_{u}^{\mu}\right) z_{u, v}^{\mu}+f_{u}^{\mu}\left(u, y_{u}^{\mu}\right) v, \quad z_{u, v}^{\mu}(0)=0
$$

and that of the mapping $(x, \mu) \mapsto p_{x}^{2, \mu}$ (recall that $p_{x}^{2, \mu}$ is the solution of (4.22) ) w.r.t. $x=$ $\left(u, \eta^{2}\right)$ in direction $h=(v, \xi)$ is the solution $\pi_{x, h}^{2, \mu}$ of (omitting the arguments $\left.\left(u, y_{u}^{\mu}, p_{x}^{2, \mu}, \eta^{2}\right)\right)$ :

$$
\begin{aligned}
-\dot{\pi}_{x, h}^{2, \mu} & =\tilde{H}_{y u}^{\mu} v+\tilde{H}_{y y}^{\mu} z_{u, v}^{\mu}+\pi_{x, h}^{2, \mu} f_{y}^{\mu}+\xi\left(g^{\mu}\right)_{y}^{(2)}, \\
\pi_{x, h}^{2, \mu}(T) & =\phi_{y y}^{\mu}\left(y_{u}^{\mu}(T)\right) z_{u, v}^{\mu}(T) .
\end{aligned}
$$

Applications of Gronwall's Lemma shows that, for $\mu$ in a neighborhood of $\bar{\mu}, x=\left(u, \eta^{2}\right)$ in a $L^{\infty}$-neighborhood of $\bar{x}=\left(\bar{u}, \bar{\eta}^{2}\right)$ and a direction $h=(v, \xi) \in X$,

$$
\begin{aligned}
& \left\|z_{u, v}^{\mu}\right\|_{\infty}=\mathcal{O}\left(\|v\|_{2}\right), \quad\left\|\pi_{x, h}^{2, \mu}\right\|_{\infty}=\mathcal{O}\left(\|h\|_{X}\right), \\
& \left\|z_{u, v}^{\mu}-z_{\bar{u}, v}^{\bar{\mu}}\right\|_{\infty}=\mathcal{O}\left(\|u-\bar{u}\|_{2}+\|\mu-\bar{\mu}\|\right)\|v\|_{2}, \\
& \left\|\pi_{x, h}^{2, \mu}-\pi_{\bar{x}, h}^{2, \bar{\mu}}\right\|_{\infty}=\mathcal{O}\left(\|x-\bar{x}\|_{X}+\|\mu-\bar{\mu}\|\right)\|h\|_{X} .
\end{aligned}
$$

In all the section, we use the following notations (time dependence is omitted):

$$
\begin{aligned}
S & :=\tilde{H}_{u u}\left(\bar{u}, \bar{y}, \bar{p}^{2}, \bar{\eta}^{2}\right), \quad R:=\tilde{H}_{u y}\left(\bar{u}, \bar{y}, \bar{p}^{2}, \bar{\eta}^{2}\right), \quad Q:=\tilde{H}_{y y}\left(\bar{u}, \bar{y}, \bar{p}^{2}, \bar{\eta}^{2}\right), \\
A & :=f_{y}(\bar{u}, \bar{y}), \quad B:=f_{u}(\bar{u}, \bar{y}), \quad \Phi:=\phi_{y y}(\bar{y}(T)), \\
C & :=g_{y}(\bar{y}), \quad d:=g(\bar{y}), \quad C_{1}=g_{y}^{(1)}(\bar{y}), \\
C_{2} & :=g_{y}^{(2)}(\bar{u}, \bar{y}), \quad N_{2}:=g_{u}^{(2)}(\bar{u}, \bar{y}), \quad a:=-C_{2} \bar{\eta}^{2}, \quad b:=-N_{2} \bar{\eta}^{2} .
\end{aligned}
$$

All the above quantities are bounded and Lipschitz continuous over $[0, T]$. By the chain rule, we obtain that

$$
D_{x} \mathcal{F}(\bar{x}, \bar{\mu})(x-\bar{x})=\left(\begin{array}{c}
S(u-\bar{u})+R z_{u-\bar{u}}+\pi_{u-\bar{u}, \eta^{2}-\bar{\eta}^{2}}^{2} B+\left(\eta^{2}-\bar{\eta}^{2}\right) N_{2} \\
C z_{u-\bar{u}}
\end{array}\right),
$$

where $z_{u-\bar{u}}:=z_{\bar{u}, u-\bar{u}}^{\bar{\mu}}$ is the solution of 5.3 for $v=u-\bar{u}$, and $\pi_{u-\bar{u}, \eta^{2}-\bar{\eta}^{2}}^{2}:=\pi_{\bar{x},(x-\bar{x})}^{2, \bar{\mu}}$ is the solution of, for $(v, \xi)=\left(u-\bar{u}, \eta^{2}-\bar{\eta}^{2}\right)$ :

$$
-\dot{\pi}_{v, \xi}^{2}=R^{\top} v+Q z_{v}+\pi_{v, \xi}^{2} A+\xi C_{2}, \quad \pi_{v, \xi}^{2}(T)=\Phi z_{v}(T) .
$$

Set $v:=u-\bar{u}$, and let $\delta=(\gamma, \zeta) \in \Delta$. Then 4.13 has a unique solution $x=\left(u, \eta^{2}\right) \in \tilde{X}$ iff the system of equations below has a unique solution $\left(v, z, \pi^{2}, \eta^{2}\right)$ with $\left(\bar{u}+v, \eta^{2}\right) \in \tilde{X}$ :

$$
\begin{aligned}
\dot{z} & =A z+B v, \quad z(0)=0, \\
-\dot{\pi}^{2} & =R^{\top} v+Q z+\pi^{2} A+\eta^{2} C_{2}-\bar{\eta}^{2} C_{2}, \quad \pi^{2}(T)=\Phi z(T) \\
0 & =S v+R z+\pi^{2} B+\eta^{2} N_{2}-\bar{\eta}^{2} N_{2}-\gamma, \\
0 & \geq d+C z-\zeta, \quad \mathrm{d} \dot{\eta}^{2} \geq 0, \quad\left\langle\mathrm{~d} \dot{\eta}^{2}, d+C z-\zeta\right\rangle=0 .
\end{aligned}
$$


We recognize the first-order necessary optimality condition of linear-quadratic problem $\left(\mathcal{P}_{\delta}\right)$ in its alternative form. That is, setting $\mathrm{d} \eta=\mathrm{d} \dot{\eta}^{2}$ and $\pi=\pi^{2}-C \dot{\eta}^{2}+C_{1} \eta^{2}$, we recover the "classical" optimality conditions of $\left(\mathcal{P}_{\delta}\right)$ (note that $C_{1}=\dot{C}+C A, C_{2}=\dot{C}_{1}+C_{1} A$, $N_{2}=C_{1} B$ and $\left.C B=g_{u}^{(1)}(\bar{u}, \bar{y}) \equiv 0\right)$ :

$$
\begin{aligned}
\dot{z} & =A z+B v, \quad z(0)=0, \\
-\mathrm{d} \dot{\pi} & =\left(R^{\top} v+Q z+\pi A-\bar{\eta}^{2} C_{2}\right) \mathrm{d} t+C \mathrm{~d} \eta, \quad \pi(T)=\Phi z(T) \\
0 & =S v+R z+\pi B-\bar{\eta}^{2} N_{2}-\gamma, \\
0 & \geq d+C z-\zeta, \quad \mathrm{d} \eta \geq 0, \quad\langle\mathrm{~d} \eta, d+C z-\zeta\rangle=0 .
\end{aligned}
$$

We see then that $(\bar{v}, \bar{z}):=0$ is a stationary point of $\left(\mathcal{P}_{0}\right)$, with alternative multipliers $\bar{\pi}^{2}:=0$ and $\bar{\eta}^{2}$, and classical multipliers $\bar{\pi}:=-C \dot{\bar{\eta}}^{2}+C_{1} \bar{\eta}^{2}$ and $\bar{\eta}=\dot{\bar{\eta}}^{2}$. The second-order optimality condition (3.6), with the quadratic cost expressed by (3.7), is precisely the condition 5.10 and implies that $(\bar{v}, \bar{z})=0$ is a local solution of $\left(\mathcal{P}_{0}\right)$.

The verifications of assumptions (iii) and (v) in Lemmas 6.1 and 6.3 are only technical, and for assumption (iv) in Lemma 6.2, we use Th. 5.4 .

Lemma 6.1. The mapping $\Psi^{\mu}=\mathcal{F}(\cdot, \mu)-\mathcal{L}(\cdot)$ is strictly stationary at $x=\bar{x}$, uniformly in $\mu$ near $\bar{\mu}$.

Proof. Let $x_{1}, x_{2} \in X$ and $\mu \in P$. We have that

$$
\begin{aligned}
\Psi^{\mu}\left(x_{1}\right)-\Psi^{\mu}\left(x_{2}\right) & =\mathcal{F}\left(x_{1}, \mu\right)-\mathcal{F}\left(x_{2}, \mu\right)-D_{x} \mathcal{F}(\bar{x}, \bar{\mu})\left(x_{1}-x_{2}\right) \\
& =\int_{0}^{1}\left(D_{x} \mathcal{F}\left(\theta x_{1}+(1-\theta) x_{2}, \mu\right)-D_{x} \mathcal{F}(\bar{x}, \bar{\mu})\right) \mathrm{d} \theta\left(x_{1}-x_{2}\right) .
\end{aligned}
$$

Let $x=\left(u, \eta^{2}\right) \in \tilde{X}$. Then by 4.17-4.18, if $x$ is close to $\bar{x}=\left(\bar{u}, \bar{\eta}^{2}\right)$ for the norm of $X$, this implies that $\left(u, \eta^{2}\right)$ belongs to a $L^{\infty}$-neighborhood of $\left(\bar{u}, \bar{\eta}^{2}\right)$. Hence, $y_{u}^{\mu}$ and $p_{u, \eta^{2}}^{2, \mu}$ remain also uniformly bounded for $\mu$ in a neighborhood of $\bar{\mu}$. Let $x_{i}=\left(u_{i}, \eta_{i}^{2}\right) \in X, i=1,2$, and given $\theta \in[0,1]$, write $x_{\theta}:=\theta x_{1}+(1-\theta) x_{2}$ and similarly for the other variables. Set

$$
\left(\begin{array}{l}
r_{1} \\
r_{2}
\end{array}\right):=\left(D_{x} \mathcal{F}\left(x_{\theta}, \mu\right)-D_{x} \mathcal{F}(\bar{x}, \bar{\mu})\right)\left(x_{1}-x_{2}\right) .
$$

Let us express the first row $r_{1}$. Omitting by $(\cdot)$ the $\operatorname{arguments}\left(u_{\theta}, y_{u_{\theta}}^{\mu}, p_{x_{\theta}}^{2, \mu}, \eta_{\theta}^{2}\right)$, we obtain that

$$
\begin{aligned}
r_{1}= & \left(\tilde{H}_{u u}^{\mu}(\cdot)-S\right)\left(u_{1}-u_{2}\right)+\left(\tilde{H}_{u y}^{\mu}(\cdot) z_{u_{\theta}, u_{1}-u_{2}}^{\mu}-R z_{\bar{u}, u_{1}-u_{2}}^{\bar{\mu}}\right) \\
& +\left(\pi_{x_{\theta}, x_{1}-x_{2}}^{2, \mu} f_{u}^{\mu}(\cdot)-\pi_{\bar{x}, x_{1}-x_{2}}^{2, \bar{c}} B\right)+\left(\eta_{1}^{2}-\eta_{2}^{2}\right)\left(\left(g^{\mu}\right)_{u}^{(2)}(\cdot)-N_{2}\right) .
\end{aligned}
$$

For $\left(u_{i}, \eta_{i}^{2}\right)$ in a $L^{\infty}$-neighborhood of $\left(\bar{u}, \bar{\eta}^{2}\right)$ and $\mu$ in the neighborhood of $\bar{\mu}$, we have that $\tilde{H}_{u u}^{\mu}(\cdot)-S=\tilde{H}_{u u}^{\mu}\left(u_{\theta}, y_{u_{\theta}}^{\mu}, p_{x_{\theta}}^{2, \mu}, \eta_{\theta}^{2}\right)-\tilde{H}_{u u}^{\bar{\mu}}\left(\bar{u}, \bar{y}, \bar{p}^{2}, \bar{\eta}^{2}\right)$ is arbitrarily small in the $L^{\infty}$ norm, 
and similarly for the terms involving the other derivatives, $\tilde{H}_{u y}^{\mu}, f_{u}^{\mu}$, and $\left(g^{\mu}\right)_{u}^{(2)}$. Therefore, given any $\varepsilon>0$, for $\left\|x_{i}-\bar{x}\right\|_{X},\|\mu-\bar{\mu}\|$ small enough,

$$
\begin{aligned}
\left\|r_{1}\right\|_{2} \leq & \varepsilon\left(\left\|u_{1}-u_{2}\right\|_{2}+\left\|z_{u_{\theta}, u_{1}-u_{2}}^{\mu}\right\|_{2}+\left\|\pi_{x_{\theta}, x_{1}-x_{2}}^{2, \mu}\right\|_{2}+\left\|\eta_{1}^{2}-\eta_{2}^{2}\right\|_{2}\right) \\
& +\|R\|_{\infty}\left\|z_{u_{\theta}, u_{1}-u_{2}}^{\mu}-z_{\bar{u}, u_{1}-u_{2}}^{\bar{\mu}}\right\|_{2}+\|B\|_{\infty}\left\|\pi_{x_{\theta}, x_{1}-x_{2}}^{2, \mu}-\pi_{\bar{x}, x_{1}-x_{2}}^{2, \bar{\mu}}\right\|_{2} .
\end{aligned}
$$

Using 6.1 -6.3 with $x=x_{\theta}$ and $h=x_{1}-x_{2}$, we obtain that $\left\|r_{1}\right\|_{2} \leq \varepsilon\left\|x_{1}-x_{2}\right\|_{X}$, whenever $x_{1}, x_{2}$ are close enough to $\bar{x}$ in $X$ and $\mu$ is close enough to $\bar{\mu}$. For the second row $r_{2}$, we have that

$$
\begin{aligned}
r_{2}= & g_{y}^{\mu}\left(y_{u_{\theta}}^{\mu}\right) z_{u_{\theta}, u_{1}-u_{2}}^{\mu}-g_{y}^{\bar{\mu}}(\bar{y}) z_{\bar{u}, u_{1}-u_{2}}^{\bar{\mu}}, \\
\dot{r}_{2}= & \left(g^{\mu}\right)_{y}^{(1)}\left(y_{u_{\theta}}^{\mu}\right) z_{u_{\theta}, u_{1}-u_{2}}^{\mu}-\left(g^{\bar{\mu}}\right)_{y}^{(1)}(\bar{y}) z_{\bar{u}, u_{1}-u_{2}}^{\bar{\mu}} \\
\ddot{r}_{2}= & \left(\left(g^{\mu}\right)_{u}^{(2)}\left(u_{\theta}, y_{u_{\theta}}^{\mu}\right)-\left(g^{\bar{\mu}}\right)_{u}^{(2)}(\bar{u}, \bar{y})\right)\left(u_{1}-u_{2}\right) \\
& +\left(g^{\mu}\right)_{y}^{(2)}\left(u_{\theta}, y_{u_{\theta}}^{\mu}\right) z_{u_{\theta}, u_{1}-u_{2}}^{\mu}-\left(g^{\bar{\mu}}\right)_{y}^{(2)}(\bar{u}, \bar{y}) z_{\bar{u}, u_{1}-u_{2}}^{\bar{\mu}} .
\end{aligned}
$$

Therefore, we conclude with the same arguments that $\left\|r_{2}\right\|_{2,2} \leq \varepsilon\left\|u_{1}-u_{2}\right\|_{2}$, whenever $\left\|x_{i}-\bar{x}\right\|_{X}, i=1,2$ and $\|\mu-\bar{\mu}\|$ are small enough. This shows the desired property.

Lemma 6.2. For $k$ sufficiently large w.r.t. $l$ in definition (4.15) of the space $X, r$ small enough in definition 4.19 of the space $\tilde{X}$, and small enough positive constants $\varrho$ and $k^{\prime}$ in definition (4.23) of the set $\Delta, 4$, has a unique solution $x_{\delta}=\left(u_{\delta}, \eta_{\delta}^{2}\right)$ in $\tilde{X}$, for all $\delta \in \Delta$, and this solution is Lipschitz continuous w.r.t. $\delta$.

Proof. We have that $x=\left(u, \eta^{2}\right)$ is solution of 4.13$)$ iff $\left(v:=u-\bar{u}, z_{v}\right)$ is solution of the first-order optimality condition of $\left(\mathcal{P}_{\delta}\right)$ with alternative multipliers $\pi_{v, \eta^{2}-\bar{\eta}^{2}}^{2}$ and $\eta^{2}$. By the hypotheses of Th. 4.3 . $(\bar{v}, \bar{z})=0$ is a stationary point of $\left(\mathcal{P}_{0}\right)$ satisfying the assumptions of Th. 5.4. Choose $\varrho$ small enough, so that $B_{W}(0, \varrho)$ is included in the neighborhood $\mathcal{W}$ of Th. 5.4. By this theorem, for all $\delta \in B_{W}(0, \varrho),\left(\mathcal{P}_{\delta}\right)$ has a unique stationary point $\left(v_{\delta}, z_{v_{\delta}}\right)$ with $\left\|v_{\delta}\right\|_{2}<\rho$ and unique associated alternative multipliers $\left(\pi_{v_{\delta}, \eta_{\delta}^{2}-\bar{\eta}^{2}}^{2}, \eta_{\delta}^{2}\right)$. Therefore, 4.13) has a unique solution $\left(u_{\delta}:=\bar{u}+v_{\delta}, \eta_{\delta}^{2}\right)$ with $\left\|u_{\delta}-\bar{u}\right\|_{2}<\rho$. We have to show that $\left(u_{\delta}, \eta_{\delta}^{2}\right)$ belongs to the space $\tilde{X}$. Throughout the proof, we denote by $C$ different positive constants.

By Prop. 5.2(i), reducing $\varrho$ if necessary, we immediately obtain that $\eta_{\delta}^{2}$ belongs to the space $B V_{T, l}^{2}[0, T]$, for large enough $l$. Therefore, by 4.18 and $\left(5.12\right.$, for all $\delta \in B_{W}(0, \varrho)$,

$$
\left\|\eta_{\delta}^{2}-\bar{\eta}^{2}\right\|_{\infty} \leq \sqrt[3]{6 l}\left\|\eta_{\delta}^{2}-\bar{\eta}^{2}\right\|_{2}^{2 / 3} \leq \sqrt[3]{6 l} \lambda^{2 / 3}\|\delta\|_{W}^{2 / 3}
$$

For $\delta=(\gamma, \zeta) \in \Delta$ (then $\left.\gamma \in \operatorname{Lip}_{k^{\prime}}\right)$, let us show now that $u_{\delta}=\bar{u}+v_{\delta} \in \operatorname{Lip}_{k}$. From the first-order alternative optimality condition of $\left(\mathcal{P}_{\delta}\right)$, we have that

$$
S v_{\delta}+R z_{v_{\delta}}+\pi_{v_{\delta}, \eta_{\delta}^{2}-\bar{\eta}^{2}}^{2} B+N_{2}\left(\eta_{\delta}^{2}-\bar{\eta}^{2}\right)-\gamma=0 .
$$


Since $S$ is uniformly invertible by (A3), using (6.1), (5.12), and (4.17), we deduce that

$$
\begin{aligned}
\left\|v_{\delta}\right\|_{\infty} & \leq C\left(\left\|z_{v_{\delta}}\right\|_{\infty}+\left\|\pi_{v_{\delta}, \eta_{\delta}^{2}-\bar{\eta}^{2}}^{2}\right\|_{\infty}+\left\|\eta_{\delta}^{2}-\bar{\eta}^{2}\right\|_{\infty}\right)+\|\gamma\|_{\infty} \\
& \leq C\left(2 \lambda\|\delta\|_{W}+\sqrt[3]{6 l} \lambda^{2 / 3}\|\delta\|_{W}^{2 / 3}\right)+\sqrt[3]{3 k^{\prime}}\|\gamma\|_{2}^{2 / 3} \\
& \leq\left(C(l)+\sqrt[3]{3 k^{\prime}}\right)\|\delta\|_{W}^{2 / 3}
\end{aligned}
$$

We denote here and in what follows by $C(l)$ different positive constants that depend on $l$ (but not on $k$ ). By time differentiation of (6.4), which is licit since $\gamma \in \operatorname{Lip}_{k^{\prime}}, \eta_{\delta}^{2}, \bar{\eta}^{2} \in$ $B V_{T, l}^{2} \subset L_{i p}, z_{v_{\delta}}, \pi_{v_{\delta}, \eta_{\delta}^{2}-\bar{\eta}^{2}}^{2} \in W^{1, \infty}, S, R, \overline{B, N_{2}}$ are Lipschitz continuous, and $S$ is uniformly invertible, it follows that

$S \dot{v}_{\delta}+\dot{S} v_{\delta}+R \dot{z}_{v_{\delta}}+\dot{R} z_{v_{\delta}}+\dot{\pi}_{v_{\delta}, \eta_{\delta}^{2}-\bar{\eta}^{2}}^{2} B+\pi_{v_{\delta}, \eta_{\delta}^{2}-\bar{\eta}^{2}}^{2} \dot{B}+N_{2}\left(\dot{\eta}^{2}-\dot{\bar{\eta}}^{2}\right)+\dot{N}_{2}\left(\eta^{2}-\bar{\eta}^{2}\right)-\dot{\gamma}=0$

Since $\left\|z_{v_{\delta}}\right\|_{\infty},\left\|\pi_{v_{\delta}, \eta_{\delta}^{2}-\bar{\eta}^{2}}^{2}\right\|_{\infty},\left\|\dot{z}_{v_{\delta}}\right\|_{\infty},\left\|\dot{\pi}_{v_{\delta}, \eta_{\delta}^{2}-\bar{\eta}^{2}}^{2}\right\|_{\infty} \leq C\left(\left\|v_{\delta}\right\|_{\infty}+\left\|\eta_{\delta}^{2}-\bar{\eta}^{2}\right\|_{\infty}\right)$, and $S$ has a bounded inverse over $[0, T]$, using that $\left\|\dot{\eta}_{\delta}^{2}\right\|_{\infty},\left\|\dot{\bar{\eta}}^{2}\right\|_{\infty} \leq l$, we obtain that

$$
\begin{aligned}
\left\|\dot{v}_{\delta}\right\|_{\infty} & \leq C\left(\left\|v_{\delta}\right\|_{\infty}+\left\|\eta_{\delta}^{2}-\bar{\eta}^{2}\right\|_{\infty}+\left\|\dot{\eta}_{\delta}^{2}-\dot{\bar{\eta}}^{2}\right\|_{\infty}\right)+\|\dot{\gamma}\|_{\infty} \\
& \leq\left(C(l)+C \sqrt[3]{3 k^{\prime}}\right)\|\delta\|_{W}^{2 / 3}+2 C l+k^{\prime} .
\end{aligned}
$$

Therefore, we have that $\left\|\dot{v}_{\delta}\right\|_{\infty} \leq k / 2$ if, fixing a suitable $l$, we take $k$ so large that $k>$ $\max \left\{4 C l ; 2\|\dot{\bar{u}}\|_{\infty}\right\}$, and choose $\varrho$ and $k^{\prime}$ in 4.23 small enough. It follows that the solution $x_{\delta}=\left(u_{\delta}=\bar{u}+v_{\delta}, \eta_{\delta}^{2}\right)$ of (4.13) belongs to the space $X$. In addition, if we choose $r=\rho$, with the $\rho$ of Th. 5.4 then $x_{\delta} \in \tilde{X}$ for $\|\delta\|_{W}$ small enough, and is the unique solution of 4.13 in $\tilde{X}$. Moreover, by Th. 5.4 .

$$
\left\|u_{\delta}-u_{\delta^{\prime}}\right\|_{2}+\left\|\eta_{\delta}^{2}-\eta_{\delta^{\prime}}^{2}\right\|_{2} \leq \lambda\left\|\delta-\delta^{\prime}\right\|_{W}, \quad \forall \delta, \delta^{\prime} \in \Delta .
$$

This achieves the proof of assumption (iv) of Th. 4.8 .

Lemma 6.3. Their exists a neighborhood of $(\bar{x}, \bar{\mu})$, such that $\mathcal{F}(x, \mu)-\mathcal{L}(x)$ belongs to $\Delta$, for all $(x, \mu)$ in this neighborhood.

Proof. We have to show that for $\|x-\bar{x}\|_{X},\|\mu-\bar{\mu}\|$ small enough, $\mathcal{F}(x, \mu)-\mathcal{L}(x) \in \Delta$, where $\Delta$ is our set of smooth variations defined by 4.23 . Throughout the proof, we denote by $C$ different positive constants. For $\theta \in[0,1]$, set $x_{\theta}:=\theta x+(1-\theta) \bar{x}$ and a similar definition of $\mu_{\theta}$. We have that

$$
\begin{aligned}
\mathcal{F}(x, \mu)-\mathcal{L}(x)= & \mathcal{F}(x, \mu)-\mathcal{F}(\bar{x}, \bar{\mu})-D_{x} \mathcal{F}(\bar{x}, \bar{\mu})(x-\bar{x}) \\
= & \int_{0}^{1}\left(D_{x} \mathcal{F}\left(x_{\theta}, \mu_{\theta}\right)-D_{x} \mathcal{F}(\bar{x}, \bar{\mu})\right) \mathrm{d} \theta(x-\bar{x}) \\
& +\int_{0}^{1} D_{\mu} \mathcal{F}\left(x_{\theta}, \mu_{\theta}\right) \mathrm{d} \theta(\mu-\bar{\mu})=:\left(\begin{array}{c}
r_{1} \\
r_{2}
\end{array}\right) .
\end{aligned}
$$


Let us show that $\left\|r_{1}\right\|_{2}+\left\|r_{2}\right\|_{2,2} \leq \varrho$ and $\left\|\dot{r}_{1}\right\|_{\infty} \leq k^{\prime}$, for $\|x-\bar{x}\|_{X}$ and $\|\mu-\bar{\mu}\|$ small enough. By the arguments of Lemma 6.1. given any $\varepsilon>0$, for $\|x-\bar{x}\|_{X}$ and $\|\mu-\bar{\mu}\|$ small enough, we have that $\left\|\int_{0}^{1}\left(D_{x} \mathcal{F}\left(x_{\theta}, \mu_{\theta}\right)-D_{x} \mathcal{F}(\bar{x}, \bar{\mu})\right) \mathrm{d} \theta(x-\bar{x})\right\|_{W} \leq \varepsilon\|x-\bar{x}\|_{X}$. Moreover, since $D_{\mu} \mathcal{F}(x, \mu)$ is uniformly bounded for $(x, \mu)$ in a neighborhood of $(\bar{x}, \bar{\mu})$ by definition of a stable extension, we deduce that

$$
\left\|r_{1}\right\|_{2}+\left\|r_{2}\right\|_{2,2} \leq \varepsilon\|x-\bar{x}\|_{X}+C\|\mu-\bar{\mu}\| \leq \varrho,
$$

for $\|x-\bar{x}\|_{X}$ and $\|\mu-\bar{\mu}\|$ small enough. Making now explicit the expression of $r_{1}$, we obtain that (recall the notations $\left.S=\tilde{H}_{u u}^{\bar{\mu}}, R=\tilde{H}_{u y}^{\bar{\mu}}, B=f_{u}^{\bar{\mu}}, N_{2}=\left(g^{\bar{\mu}}\right)_{u}^{(2)}\right)$ :

$$
\begin{aligned}
r_{1}= & \tilde{H}_{u}^{\mu}\left(u, y_{u}^{\mu}, p_{u, \eta^{2}}^{2, \mu}, \eta^{2}\right)-\tilde{H}_{u}^{\bar{\mu}}\left(\bar{u}, \bar{y}, \bar{p}^{2}, \bar{\eta}^{2}\right)-S(u-\bar{u})-R z_{u-\bar{u}} \\
& -\pi_{u-\bar{u}, \eta^{2}-\bar{\eta}^{2}}^{2} B-N_{2}\left(\eta^{2}-\bar{\eta}^{2}\right) .
\end{aligned}
$$

Time derivation yields (omitting arguments and reorganizing the terms)

$$
\begin{aligned}
\dot{r}_{1}= & \left(\tilde{H}_{u u}^{\mu}-\tilde{H}_{u u}^{\bar{\mu}}\right) \dot{u}+\left(\tilde{H}_{u y}^{\mu} f^{\mu}-\tilde{H}_{u y}^{\bar{\mu}} f^{\bar{\mu}}\right)-\left(\tilde{H}_{y}^{\mu} f_{u}^{\mu}-\tilde{H}_{y}^{\bar{\mu}} f_{u}^{\bar{\mu}}\right)+\left(\left(g^{\mu}\right)_{u}^{(2)}-\left(g^{\bar{\mu}}\right)_{u}^{(2)}\right) \dot{\eta}^{2} \\
& -R \dot{z}_{u-\bar{u}}-\dot{\pi}_{u-\bar{u}, \eta^{2}-\bar{\eta}^{2}}^{2} B-\dot{S}(u-\bar{u})-\dot{R} z_{u-\bar{u}}-\pi_{u-\bar{u}, \eta^{2}-\bar{\eta}^{2}}^{2} \dot{B}-\dot{N}_{2}\left(\eta^{2}-\bar{\eta}^{2}\right) .
\end{aligned}
$$

For $\left(u, \eta^{2}\right)$ close to $\left(\bar{u}, \bar{\eta}^{2}\right)$ in $X$, and $\mu$ in a neighborhood of $\bar{\mu}$, we have by (4.17)-(4.18) that $\left\|\left(u, y_{u}^{\mu}, p_{u, \eta^{2}}^{2, \mu}, \eta^{2}\right)-\left(\bar{u}, \bar{y}, \bar{p}^{2}, \bar{\eta}^{2}\right)\right\|_{\infty}$ is arbitrarily small, and hence, by continuity of $H_{u u}^{\mu}$, etc, given any $\varepsilon>0$, we obtain that

$$
\begin{aligned}
\left\|\dot{r}_{1}\right\|_{\infty} \leq & \varepsilon\left(\|\dot{u}\|_{\infty}+\left\|\dot{\eta}^{2}\right\|_{\infty}+1\right)+C\left(\left\|\dot{z}_{u-\bar{u}}\right\|_{\infty}+\left\|\dot{\pi}_{u-\bar{u}, \eta^{2}-\bar{\eta}^{2}}^{2}\right\|_{\infty}\right) \\
& +C\left(\|u-\bar{u}\|_{\infty}+\left\|z_{u-\bar{u}}\right\|_{\infty}+\left\|\pi_{u-\bar{u}, \eta^{2}-\bar{\eta}^{2}}^{2}\right\|_{\infty}+\left\|\eta^{2}-\bar{\eta}^{2}\right\|_{\infty}\right) \\
\leq & \varepsilon(k+l+1)+C\left(\|u-\bar{u}\|_{\infty}+\left\|\eta^{2}-\bar{\eta}^{2}\right\|_{\infty}\right) \\
\leq & \varepsilon(k+l+1)+C(\sqrt[3]{6 k}+\sqrt[3]{6 l})\|x-\bar{x}\|_{X}^{2 / 3} \leq k^{\prime}
\end{aligned}
$$

if $\|x-\bar{x}\|_{X}$ and $\|\mu-\bar{\mu}\|$ are small enough. It follows that $r_{1} \in \operatorname{Lip}_{k^{\prime}}\left(0, T ; \mathbb{R}^{m}\right)$, and with (6.5), this achieves the proof.

Proof of Th. 4.3. We apply Th. 4.8 with the spaces $X, \tilde{X}, W, \Delta, P$ and mappings $\mathcal{F}$, $\mathcal{N}, \mathcal{L}$ defined in subsection 4.3 . We set $\bar{x}:=\left(\bar{u}, \bar{\eta}^{2}\right)$. The assumptions (i) and (ii) of Th. 4.8 are obviously fulfilled from our hypotheses and the definition of a stable extension. For an appropriate choice of the constants $k, l, r, k^{\prime}, \varrho$ involved in the definition of the spaces $X, \tilde{X}$ and $\Delta$, assumptions (iii), (iv) and (v) hold by respectively Lemmas 6.1 6.2 and 6.3 It follows that for all $\mu$ in a neighborhood of $\bar{\mu}$, there exists a unique stationary point $\left(u^{\mu}, y^{\mu}\right)$ of $\left(\mathcal{P}^{\mu}\right)$ and unique associated alternative multipliers $\left(p^{2, \mu}, \eta^{2, \mu}\right)$ with $\left(u^{\mu}, \eta^{2, \mu}\right)$ in a $X$-neighborhood of $\bar{x}$, and 4.14 is satisfied. Since by definition of a stable extension, $\mathcal{F}$ is Lipschitz continuous w.r.t. $\mu$, uniformly w.r.t. $x$, this implies that (4.2) holds, while (4.3) follows from (4.17)-(4.18). Finally, by (4.3), taking if necessary a smaller neighborhood of $\bar{\mu}$, $u^{\mu}$ belongs to the $L^{\infty}$-neighborhood of $\bar{u}$ on which the uniform quadratic growth condition holds (Prop. 4.2). Therefore, $\left(u^{\mu}, y^{\mu}\right)$ is the unique stationary point of $\left(\mathcal{P}^{\mu}\right)$ with $u^{\mu}$ in a $L^{\infty}$-neighborhood of $\bar{u}$ and is a local solution of $\left(\mathcal{P}^{\mu}\right)$ satisfying 4.1 . 


\section{Conclusion and Remarks}

In this paper, we obtain for the first time stability results for optimal control problems with a state constraint of order greater than one without any assumption on the structure of the contact set. For this we use a generalized implicit function theorem in metric spaces 9 ] applied to a system equivalent to the first-order optimality condition, involving alternative multipliers obtained by integrating the original state constraint multiplier. In the stability analysis of linear-quadratic problems, we use [6, Th. 5.17] to obtain the existence of a stationary point for the perturbed problem under a weak second-order sufficient condition taking into account the active constraints. In this way the method for weakening the secondorder sufficient condition is different from the method used in [21, 20].

Due to the low regularity of state constraint multipliers, we use a framework that differs from the ones used for first-order state constraints in [18] or in [9] in the choice of the spaces for the state constraint and state constraint multiplier. We keep the idea of [9] to use as control space the space of Lipschitz continuous functions with a bound on the Lipschitz constant.

Though the analysis is restricted to a scalar state constraint of second-order, the framework and results presented in this paper have a natural extension to several state constraints of orders $\geq 2$ (see Remarks 2.1 and 2.2). Taking into account both components of first-order and higher-order is more delicate since then the arguments used in [18, 9, 20] and in the present paper would have to be combined.

Making additional assumptions on the structure of the contact set, $L^{\infty}$ Lipschitz stability of solutions can be obtained, see [22, 5], improving (4.3), as it is the case for first-order state constraints (see [9, Section 4]). In [22, 5] it was also shown using a shooting approach that the solutions are directionally differentiable w.r.t. the parameter. It would be interesting as well to obtain sensitivity results without assumption on the structure of the contact set, extending to higher-order state constraints the sensivity results obtained by Malanowski [18] for state constraints of first-order.

Finally, let us note that the second-order sufficient condition (3.6) used in the stability analysis might be weakened by taking into account the curvature term of the constraint (see [2, Th. 27], [3, Th. 6.1] and [5, Th. 4.3]).

\section{References}

[1] L. Ambrosio, N. Fusco, and D. Pallara. Functions of bounded variation and free discontinuity problems. Oxford Mathematical Monographs. The Clarendon Press Oxford University Press, New York, 2000.

[2] J.F. Bonnans and A. Hermant. No gap second order optimality conditions for optimal control problems with a single state constraint and control. Mathematical Programming, Ser. B (to appear). 
[3] J.F. Bonnans and A. Hermant. Second-order analysis for optimal control problems with pure and mixed state constraints. INRIA Research Report 6199, 2007.

[4] J.F. Bonnans and A. Hermant. Stability and sensitivity analysis for optimal control problems with a first-order state constraint and application to continuation methods. ESAIM. Control, Optimisation and Calculus of Variations (to appear).

[5] J.F. Bonnans and A. Hermant. Well-posedness of the shooting algorithm for state constrained optimal control problems with a single constraint and control. SIAM Journal on Control and Optimization, 46(4):1398-1430, 2007.

[6] J.F. Bonnans and A. Shapiro. Perturbation analysis of optimization problems. SpringerVerlag, New York, 2000.

[7] B. Bonnard, L. Faubourg, and E. Trélat. Optimal control of the atmospheric arc of a space shuttle and numerical simulations with multiple-shooting method. Mathematical Models $\&$ Methods in Applied Sciences, 15(1):109-140, 2005.

[8] A.L. Dontchev and W. Hager. Lipschitzian stability in nonlinear control and optimization. SIAM Journal on Control and Optimization, 31(3):569-603, 1993.

[9] A.L. Dontchev and W.W. Hager. Lipschitzian stability for state constrained nonlinear optimal control. SIAM Journal on Control and Optimization, 36(2):698-718 (electronic), 1998.

[10] A.L. Dontchev and W.W. Hager. The Euler approximation in state constrained optimal control. Mathematics of Computation, 70:173-203, 2001.

[11] A.L. Dontchev, W.W. Hager, A.B. Poore, and B. Yang. Optimality, stability, and convergence in nonlinear control. Applied Mathematics and Optimization, 31(3):297$326,1995$.

[12] N. Dunford and J. Schwartz. Linear operators, Vol I and II. Interscience, New York, 1958, 1963.

[13] I. Ekeland. Nonconvex minimization problems. Bulletin of the American Mathematical Society, 1(New series):443-474, 1979.

[14] W.W. Hager. Lipschitz continuity for constrained processes. Society for Industrial and Applied Mathematics. Journal on Control and Optimization, 17(3):321-338, 1979.

[15] R.F. Hartl, S.P. Sethi, and R.G. Vickson. A survey of the maximum principles for optimal control problems with state constraints. SIAM Review, 37:181-218, 1995.

[16] A.D. Ioffe and V.M. Tihomirov. Theory of Extremal Problems. North-Holland Publishing Company, Amsterdam, 1979. Russian Edition: Nauka, Moscow, 1974.

$\mathrm{RR} \mathrm{n}^{\circ} 0123456789$ 
[17] K. Malanowski. Two-norm approach in stability and sensitivity analysis of optimization and optimal control problems. Advances in Mathematical Sciences and Applications, 2:397-443, 1993.

[18] K. Malanowski. Stability and sensitivity of solutions to nonlinear optimal control problems. Journal of Applied Mathematics \&3 Optimization, 32:111-141, 1995.

[19] K. Malanowski. Stability and sensitivity analysis for optimal control problems with control-state constraints. Dissertationes Math. (Rozprawy Mat.), 394:51, 2001.

[20] K. Malanowski. Stability analysis for nonlinear optimal control problems subject to state constraints. SIAM Journal on Optimization, 18(3):926-945, 2007.

[21] K. Malanowski. Sufficient optimality conditions in stability analysis for stateconstrained optimal control. Applied Mathematics and Optimization, 55(2):255-271, 2007.

[22] K. Malanowski and H. Maurer. Sensitivity analysis for optimal control problems subject to higher order state constraints. Annals of Operations Research, 101:43-73, 2001. Optimization with data perturbations, II.

[23] H. Maurer. On the minimum principle for optimal control problems with state constraints. Schriftenreihe des Rechenzentrum 41, Universität Münster, 1979.

[24] H. Maurer. First and second order sufficient optimality conditions in mathematical programming and optimal control. Math. Programming Stud., (14):163-177, 1981.

[25] S.M. Robinson. First order conditions for general nonlinear optimization. SIAM Journal on Applied Mathematics, 30:597-607, 1976.

[26] S.M. Robinson. Stability theorems for systems of inequalities, part II: Differentiable nonlinear systems. SIAM Journal on Numerical Analysis, 13:497-513, 1976.

[27] S.M. Robinson. Strongly regular generalized equations. Mathematics of Operations Research, 5:43-62, 1980. 


\section{Contents}

\begin{tabular}{lll}
\hline 1 & Introduction & 3
\end{tabular}

2 Preliminaries 4

2.1 Optimality conditions and Assumptions . . . . . . . . . . . . . . . . . 5

2.2 Perturbed optimal control problem . . . . . . . . . . . . . . . . . . 9

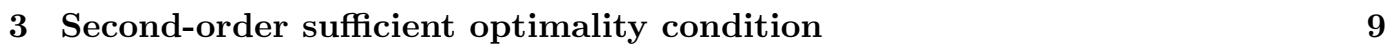

$4 \quad$ Stability analysis for the nonlinear problem 11

4.1 Stability of multipliers $\ldots \ldots \ldots \ldots \ldots \ldots$

\begin{tabular}{|ll|l|ll|}
\hline 4.2 & The uniform second-order growth condition (proof of Prop. & 4.2 & . . . . . . . . . 14 \\
\hline
\end{tabular}

4.3 The strong regularity framework $\ldots \ldots \ldots \ldots \ldots$

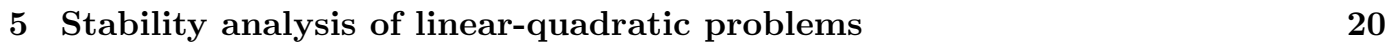

6 Proof of Theorem $|4.3|-24$

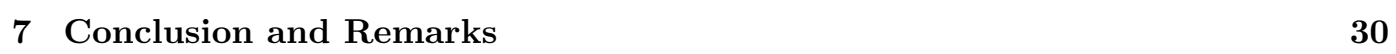

$\mathrm{RR} \mathrm{n}^{\circ} 0123456789$ 
Unité de recherche INRIA Futurs

Parc Club Orsay Université - ZAC des Vignes

4, rue Jacques Monod - 91893 ORSAY Cedex (France)

Unité de recherche INRIA Lorraine : LORIA, Technopôle de Nancy-Brabois - Campus scientifique 615, rue du Jardin Botanique - BP 101 - 54602 Villers-lès-Nancy Cedex (France)

Unité de recherche INRIA Rennes : IRISA, Campus universitaire de Beaulieu - 35042 Rennes Cedex (France)

Unité de recherche INRIA Rhône-Alpes : 655, avenue de l’Europe - 38334 Montbonnot Saint-Ismier (France)

Unité de recherche INRIA Rocquencourt : Domaine de Voluceau - Rocquencourt - BP 105 - 78153 Le Chesnay Cedex (France)

Unité de recherche INRIA Sophia Antipolis : 2004, route des Lucioles - BP 93 - 06902 Sophia Antipolis Cedex (France) 